ARTICLE

DOI: $10.1038 / s 41467-017-00547-y$

\title{
The Candida albicans transcription factor Cas5 couples stress responses, drug resistance and cell cycle regulation
}

Jinglin L. Xie (1) 1, Longguang Qin², Zhengqiang Miao², Ben T. Grys (D) 1,3, Jacinto De La Cruz Diaz ${ }^{4}$, Kenneth Ting ${ }^{1}$, Jonathan R. Krieger ${ }^{5}$, Jiefei Tong ${ }^{6}$, Kaeling Tan ${ }^{2}$, Michelle D. Leach ${ }^{1,7}$, Troy Ketela ${ }^{1}$, Michael F. Moran ${ }^{1,5,6}$, Damian J. Krysan 4,8, Charles Boone ${ }^{1,3}$, Brenda J. Andrews ${ }^{1,3}$, Anna Selmecki ${ }^{9}$, Koon Ho Wong ${ }^{2}$, Nicole Robbins ${ }^{1} \&$ Leah E. Cowen ${ }^{1}$

The capacity to coordinate environmental sensing with initiation of cellular responses underpins microbial survival and is crucial for virulence and stress responses in microbial pathogens. Here we define circuitry that enables the fungal pathogen Candida albicans to couple cell cycle dynamics with responses to cell wall stress induced by echinocandins, a front-line class of antifungal drugs. We discover that the $C$. albicans transcription factor Cas 5 is crucial for proper cell cycle dynamics and responses to echinocandins, which inhibit $\beta-1,3$-glucan synthesis. Cas5 has distinct transcriptional targets under basal and stress conditions, is activated by the phosphatase Glc7, and can regulate the expression of target genes in concert with the transcriptional regulators Swi4 and Swi6. Thus, we illuminate a mechanism of transcriptional control that couples cell wall integrity with cell cycle regulation, and uncover circuitry governing antifungal drug resistance.

\footnotetext{
${ }^{1}$ Department of Molecular Genetics, University of Toronto, Toronto, ON, Canada M5G 1M1. ${ }^{2}$ Faculty of Health Sciences, University of Macau, Macau SAR 999078, China. ${ }^{3}$ Donnelly Centre for Cellular and Biomolecular Research, University of Toronto, Toronto, ON, Canada M5S 3E1. ${ }^{4}$ Department of Microbiology and Immunology, University of Rochester, Rochester, NY 14642, USA. ${ }^{5}$ The Hospital for Sick Children, SPARC Biocentre, Toronto, ON, Canada M5G 0A4. ${ }^{6}$ The Hospital for Sick Children, Program in Cell Biology, Peter Gilgan Centre for Research and Learning, Toronto, ON, Canada M5G OA4. ${ }^{7}$ Aberdeen Fungal Group, School of Medical Sciences, Institute of Medical Sciences, University of Aberdeen, Foresterhill, Abderdeen AB252ZD, UK.

${ }^{8}$ Department of Pediatrics and Microbiology/Immunology, University of Rochester, Rochester, NY 14642, USA. ${ }^{9}$ Department of Medical Microbiology and Immunology, Creighton University School of Medicine, Omaha, NE 68178, USA. Correspondence and requests for materials should be addressed to L.E.C. (email: leah.cowen@utoronto.ca)
} 
T he fungal kingdom encompasses diverse species, including a minority that have a devastating impact on human health. One of the most pervasive fungal pathogens of humans is Candida albicans ${ }^{1,2}$, which is a commensal on the skin and mucosal surfaces of up to $60 \%$ of healthy individuals ${ }^{3}$. As an opportunist, C. albicans can exploit a decline in host immunity or an imbalance in the host microbiome, leading to diverse pathologies such as oral thrush, vaginal candidiasis, or life-threatening bloodstream infections with mortality rates of $\sim 40 \% 4,5$. C. albicans thrives as a human pathogen in part due to its ability to evade host immunity by switching between yeast and filamentous morphologies, as well as due to its capacity to withstand the hostile host environment by activating robust stress responses ${ }^{6}$. The emerging paradigm is that $C$. albicans stress response pathways are not only critical for adaptation to host conditions, but they also enable fungal virulence and drug resistance ${ }^{7-11}$.

The emergence of resistance to the limited arsenal of antifungal drugs impedes the effective treatment of systemic infections ${ }^{12-14}$. A poignant example is the evolution of resistance to the only new class of antifungal to be approved in decades, the echinocandins ${ }^{15,16}$. Echinocandins block $\beta$-1,3-glucan biosynthesis in the fungal cell wall via inhibition of the glucan synthase Fks1, thereby compromising cell wall integrity. The most common mechanism of echinocandin resistance involves mutations in the drug target $F K S 1^{17}$, 18; however, resistance phenotypes are also modulated by cellular stress responses $8,9,11$. Targeting core hubs in cellular circuitry that control responses to stress, such as the molecular chaperone Hsp90 or protein phosphatase calcineurin, has emerged as a powerful strategy to enhance antifungal activity against diverse fungal pathogens, 16 . A challenge in targeting Hsp90 or calcineurin in antifungal drug development is that they are conserved and important regulators in the human host.

An expanded repertoire of potential targets is provided by effectors downstream of these cellular regulators. C. albicans mobilizes diverse stress response programs through the action of transcription factors. For example, in response to cell membrane and cell wall stress, the transcription factor Crzl is activated by calcineurin, leading to the induction of calcineurin-dependent genes $^{19,20}$. Another example from the model yeast Saccharomyces cerevisiae is the cell wall stress-dependent activation of the transcription factor Rlm1 by the MAP kinase $\mathrm{Mpk}^{21}$. Although $\mathrm{Rlm} 1$ is the main transcriptional regulator of cell wall stress responses in S. cerevisiae, its function is not conserved in C. albicans ${ }^{22}$. In C. albicans, the zinc finger transcription factor, Cas5, serves as a key transcriptional regulator of responses to cell wall stress ${ }^{22}$. Cas5 lacks an ortholog in S. cerevisiae and most other eukaryotes, and the mechanism by which it is regulated remains enigmatic.

Activation of stress responses can induce diverse physiological changes, including modulation of cell cycle progression and remodeling of cell wall architecture ${ }^{23-27}$. The most well characterized stress response pathway involved in cell cycle regulation is controlled by the MAP kinase $\operatorname{Hog} 1^{26}$. In response to osmotic stress, Hog1 mediates a transient cell cycle arrest to enable cellular adaptation ${ }^{26}$. Multiple stress response pathways coordinate cell wall remodeling in response to environmental perturbations, including heat shock ${ }^{27}$, osmotic stress ${ }^{28}$, and cell wall stress ${ }^{29}$. However, little is known about whether cell cycle progression and cell wall remodeling are coordinated in response to stress in C. albicans, and whether there is a central signaling pathway that integrates these fundamental aspects of cellular biology.

Here, we harness genome-wide approaches to elucidate the mechanism by which Cas 5 orchestrates transcriptional changes in response to cell wall perturbation. We identify an unexpected role for Cas5 in coupling cell cycle dynamics to cell wall stress responses, and determine that Cas5 regulates distinct transcriptional programs under basal and stress conditions. We discover that Cas5 is activated by the type I protein phosphatase Glc7 in response to cell wall stress, and it regulates cell wall homeostasis in part through its interaction with Swi4-Swi6 cell cycle boxbinding factor (SBF) complex members, Swi4 and Swi6. Finally, we describe a role for Cas5 in orchestrating nuclear division. Our work provides insight into cellular reprogramming in response to cell wall stress, and establishes regulatory circuitry that couples stress response, cell cycle regulation, and drug resistance.

\section{Results}

Cas5 is a biologically responsive transcriptional regulator. Cas5 was originally identified in a genetic screen for transcription factor mutants that are hypersensitive to the echinocandin caspofungin, and microarray analysis implicated Cas5 in the regulation of genes important for cell wall integrity in response to cell wall stress ${ }^{22}$. However, the role of Cas5 under basal conditions remained elusive. To further understand Cas5 function under basal conditions, we sought to explore the impact of Cas5 on regulation of global transcriptional profiles in rich medium. To do so, we used chromatin immunoprecipitation (ChIP) of RNA polymerase II (PolII) coupled to sequencing (ChIP-Seq) with a wild-type strain and cas $5 \Delta / \operatorname{cas} 5 \Delta$ mutant. RNA PolII occupancy has been found to serve as a more precise measure of transcriptional regulation than steady state transcript levels, given that transcript levels depend on RNA turnover in addition to transcription factor activity and RNA PolII-mediated transcrip$\operatorname{tion}^{30}$. As RNA PolII recruitment corresponds to transcriptional activity, an increase in RNA PolII occupancy indicates upregulation of gene expression, whereas a decrease in RNA PolII occupancy indicates downregulation ${ }^{30,} 31$. Further, strong correlation in RNA PolII binding between our biological replicates supported the reproducibility of this method (Supplementary Fig. 1). We identified 329 genes that had increased RNA PolII occupancy, and 275 genes that had reduced PolII occupancy in the cas $5 \Delta / \operatorname{cas} 5 \Delta$ mutant as compared with the wild-type strain under standard growth conditions (Supplementary Data 1). To identify the physiological roles of Cas5, we subjected these gene sets to pathway analysis using Gene Ontology (GO). The sets that had reduced RNA PolII occupancy upon deletion of CAS5 were significantly enriched in genes with functions in diverse processes, including metabolic processes and interaction with host (Fig. 1a and Supplementary Data 1). In contrast, the gene set that had increased RNA PolII occupancy in a cas $5 \Delta /$ cas $5 \Delta$ mutant was enriched in genes with functions associated with rRNA processing, respiration, and amino acid biogenesis (Fig. 1a and Supplementary Data 1). We also employed Kyoto Encyclopedia of Genes and Genomes (KEGG) analysis as a secondary approach to identify genes and pathways affected by deletion of CAS5. Consistent with our GO term analysis, many genes with RNA PolII-binding profiles that were affected by Cas5 were associated with ribosome biogenesis and metabolic pathways (Fig. $1 \mathrm{~b}$ and Supplementary Data 1). KEGG analysis also identified a signature of differentially bound genes with functions in meiosis, cell cycle, and DNA replication (Fig. 1c and Supplementary Data 1). Thus, Cas5 governs diverse biological responses under basal conditions.

Next, we explored the impact of Cas5 on global responses to cell wall stress. We mapped genome-wide RNA PolII occupancy during caspofungin treatment in wild-type and cas $5 \Delta / \operatorname{cas} 5 \Delta$ strains. In a wild-type strain, 546 genes had distinct RNA PolIIbinding profiles in response to caspofungin treatment, with increased binding for 294 genes and reduced binding for 252 genes (Supplementary Data 2). As expected, many genes with 
a

Enriched GO process terms

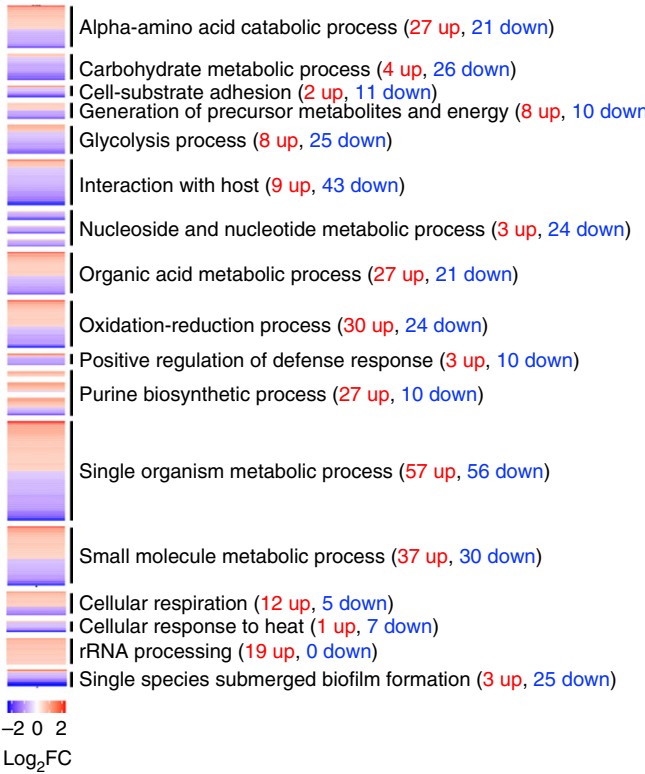

C

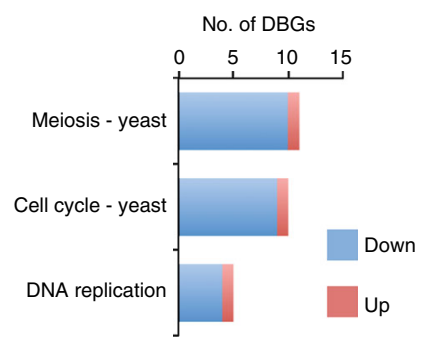

e

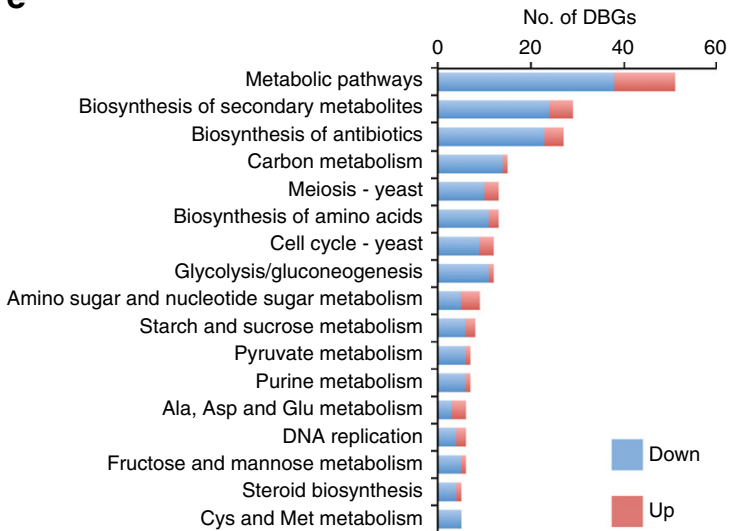

b

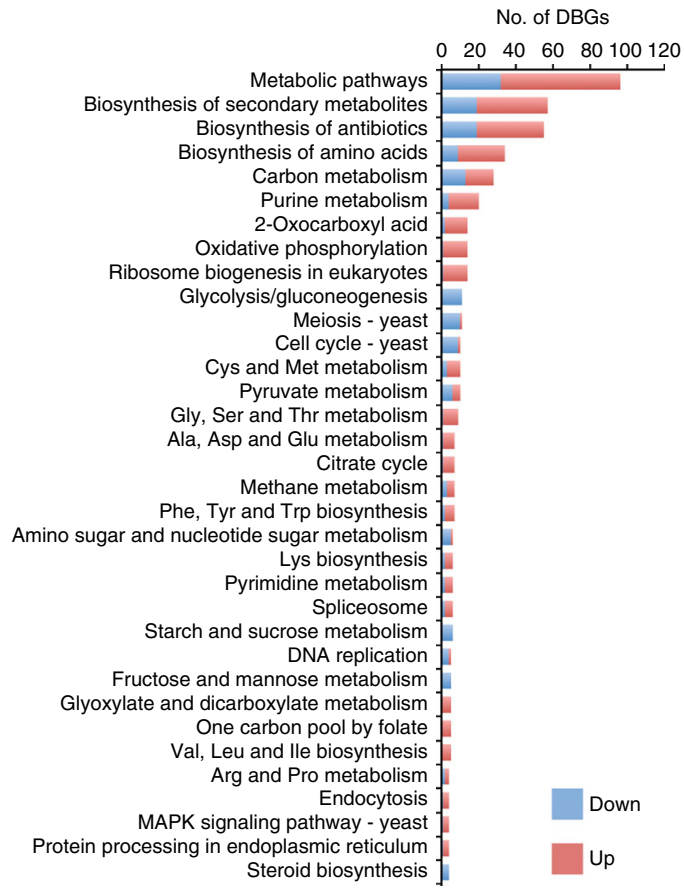

d

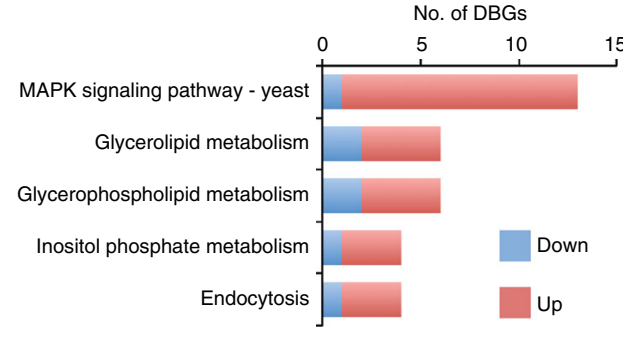

f

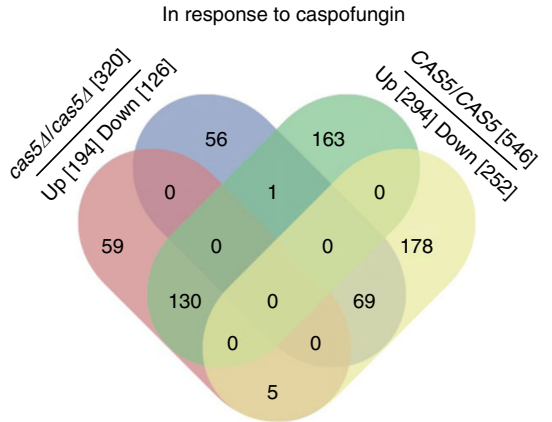

Fig. 1 Cas 5 has a profound impact on RNA Polll binding at genes implicated in cell wall and cell cycle processes under both basal and cell wall stress conditions. a Heat maps showing the genes with increased (red) and decreased (blue) RNA Polll binding in a cas $5 \Delta / \operatorname{cas} 5 \Delta$ mutant relative to wildtype. Enriched GO processes are indicated, and were clustered using the DAVID Gene Functional Classification Tool. $\mathbf{b}$ Bar chart showing the number of genes differentially bound by PollI (DBGs differentially bound genes), with increased binding in red and decreased binding in blue, in a cas $5 \Delta /$ cas $5 \Delta$ mutant relative to wildtype based on their assigned KEGG pathways. KEGG pathways with four or more genes assigned are shown. $\mathbf{c}$ Bar chart showing the number of genes differentially bound by Polll, with increased binding in red and decreased binding in blue, in a cas $5 \Delta / \operatorname{cas} 5 \Delta$ mutant relative to wildtype belonging to select KEGG pathways involved in cell cycle and related processes. d, e Bar charts showing the number of differentially bound genes involved in the indicated physiological pathways upon caspofungin treatment, with increased binding in red and decreased binding in blue, in a wild-type strain in response to caspofungin. KEGG pathways were grouped according to the ratio of genes with increased Polll binding to genes with decreased Polll binding in response to caspofungin, with $\mathbf{d}$ enriched for genes with increased Polll binding and e enriched for genes with decreased Polll binding. KEGG pathways with five or more genes assigned are shown. $\mathbf{f}$ Venn diagram depicting number of genes differentially bound by RNA Polll in response to caspofungin in the wild-type reference (increased, green; decreased, yellow) and cas $5 \Delta /$ cas $5 \Delta$ mutant (increased, red; decreased, blue) strains. See Supplementary Data 1-4 for full data sets 
increased RNA PolII binding upon caspofungin exposure were important for tolerating cell wall stress (Fig. 1d and Supplementary Data 2), whereas genes with reduced RNA PolII binding were involved in diverse biological processes, including cell cycle progression (Fig. 1e and Supplementary Data 2). Strikingly, comparison of genes that were differentially bound in response to caspofungin between the wild-type strain and the cas $5 \Delta /$ cas $5 \Delta$ mutant, revealed that $>60 \%$ of caspofungin-responsive genes were dependent on Cas5. Specifically, 163 of the 294 genes with increased PolII occupancy in response to caspofungin exposure and 178 of the 252 genes with reduced occupancy were dependent on Cas5 (Fig. 1f and Supplementary Data 2-4). These findings
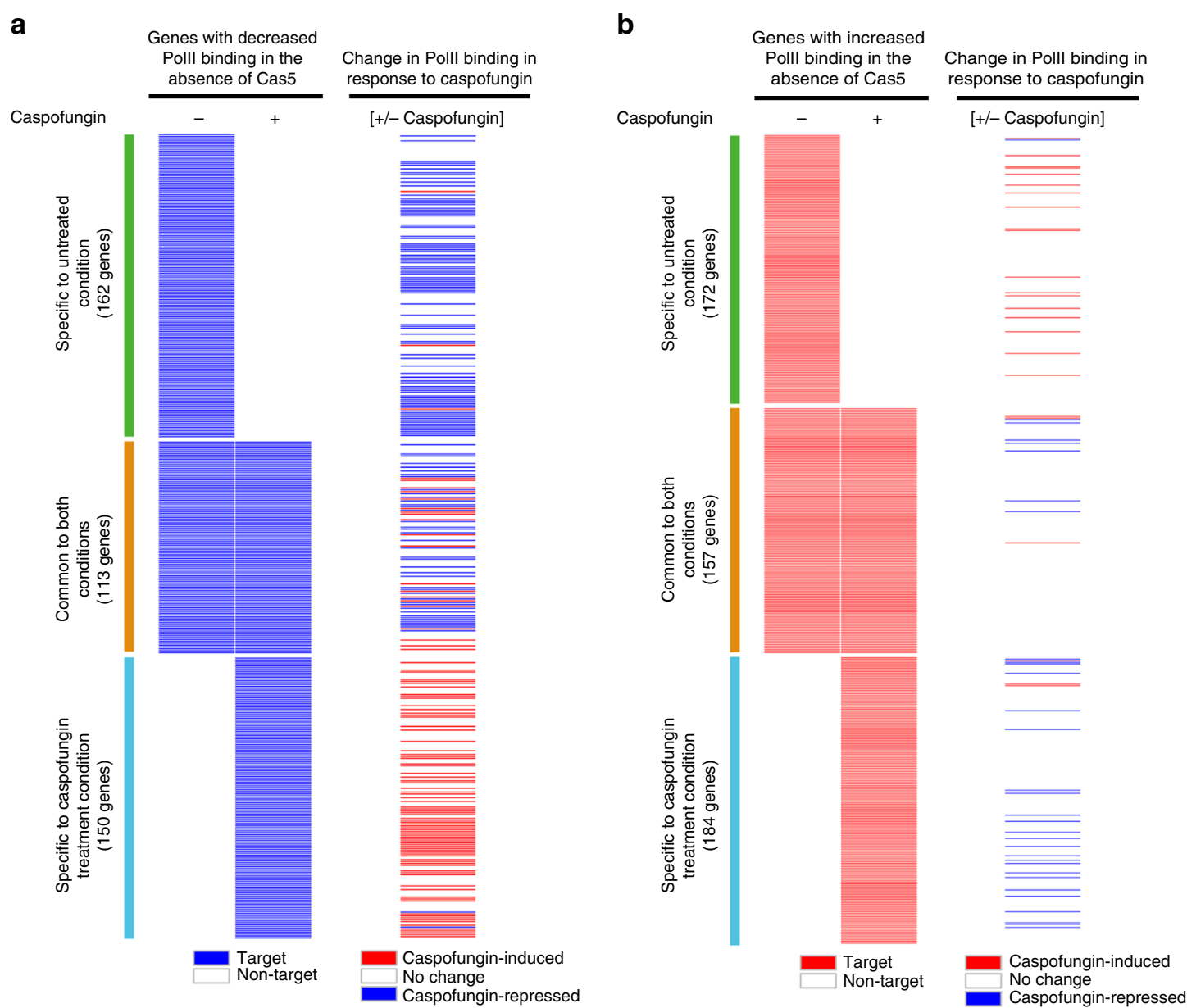

C

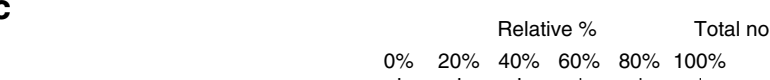

d
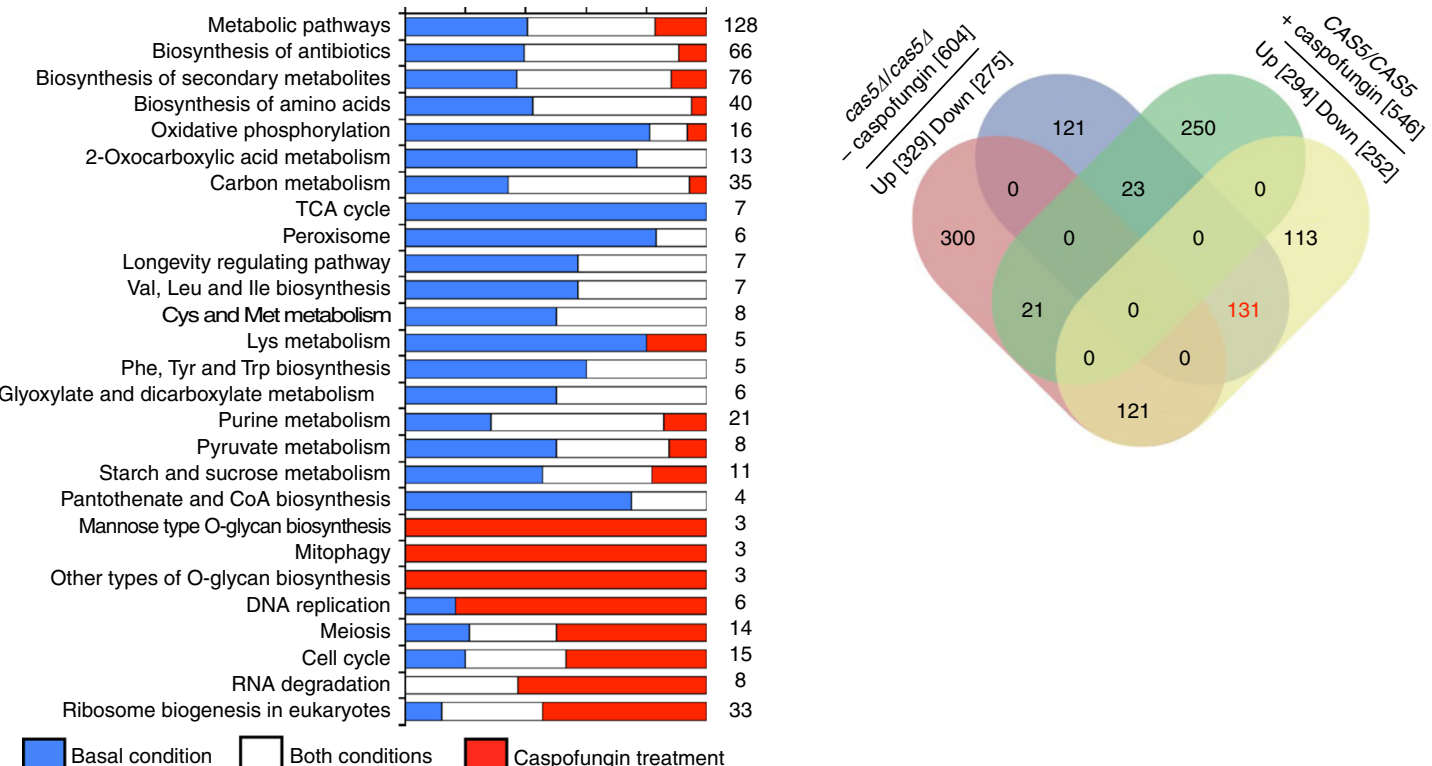
suggest that Cas5 has a profound impact on global transcriptional responses to cell wall stress.

Finally, we focused on those genes with Cas5-dependent differences in RNA PolII binding under basal and cell wall stress conditions. Strikingly, only $28 \%$ of genes with Cas5-dependent differences in RNA PolII binding were common to both untreated and caspofungin treatment conditions (Fig. 2a and Supplementary Data 1, 3 and 4). The Cas5-dependent genes specific to each condition had different physiological functions (Fig. 2c and Supplementary Data 4). Our analysis revealed a major overlap of genes that had Cas5-dependent increased RNA PolII occupancy under basal conditions with those that had reduced RNA PolII occupancy in a wild-type strain in response to caspofungin treatment (Fig. 2d and Supplementary Data 5), suggesting that caspofungin impedes Cas5-mediated expression of its basal-specific targets. Collectively, we identified hundreds of new Cas5-dependent transcriptional events under basal and stress conditions, implicating Cas5 in governing distinct transcriptional programs in response to different environmental conditions.

Cas5 is a transcriptional regulator of cell wall homeostasis. Given that Cas5 is known to regulate cell wall integrity ${ }^{22}$, we sought to further evaluate the impact of Cas5 on cell wall homeostasis. We observed that Cas5 has a profound impact on RNA PolII binding to genes with cell wall functions; GO terms from process ontology revealed that 23 of the 163 genes with Cas5-dependent increases in RNA PolII binding in response to caspofungin encode proteins involved in cell wall organization and biogenesis ( $P$-value $7.45 \mathrm{e}^{-06}$, Bonferroni Correction) (Supplementary Data 3). Among these genes were WSC1 and WSC2, which encode transmembrane sensors that respond to cell wall perturbations, yet have not been previously linked to Cas5. We confirmed the induction of these transcripts in response to caspofungin was dependent on Cas5 by quantitative RT-PCR (qRT-PCR) (Fig. 3a). We next evaluated the impact of Cas5 on tolerating cell wall stress in a wild-type strain and an echinocandin-resistant mutant. We measured susceptibility of a cas $5 \Delta / \operatorname{cas} 5 \Delta$ mutant to two cell wall perturbing agents, caspofungin and calcofluor white. The cas $5 \Delta / \operatorname{cas} 5 \Delta$ mutant was hypersensitive to both cell wall stressors, and the hypersensitivity phenotype was complemented by re-introduction of a wild-type allele (Fig. 3b). Given that the most common mechanism of resistance to echinocandins is mutations in the drug target gene, FKS1, we tested whether Cas5 also enables Fks-dependent caspofungin resistance. Homozygous deletion of CAS5 reduced resistance of a strain carrying the Fks $1^{\text {F641S }}$ substitution, suggesting Cas 5 is a key regulator of target-mediated echinocandin resistance (Fig. 3b). Thus, Cas5 regulates cell wall stability during periods of cell wall stress, with a profound impact on drug resistance.
Next, we assessed if Cas5 regulates the expression of genes important for cell wall homeostasis in the absence of cell wall stress. We found that 110 out of the 275 genes that have reduced RNA PolII binding in the cas $5 \Delta / \operatorname{cas} 5 \Delta$ mutant under basal conditions were associated with cell membrane, cell wall, and cell periphery GO components (Supplementary Data 1). Thus, we predicted Cas 5 enables cell wall stability in the absence of cell wall stress, such that deletion of CAS5 would induce cell wall stress under basal conditions. To determine if a $\operatorname{cas} 5 \Delta / \operatorname{cas} 5 \Delta$ mutant is intrinsically defective in cell wall architecture, we monitored phosphorylation of the terminal cell wall integrity MAP kinase, $\mathrm{Mkc1}$, under basal conditions ${ }^{32}$. Compared with a wild-type control, the cas $5 \Delta / \operatorname{cas} 5 \Delta$ mutant had elevated levels of Mkc1 phosphorylation under basal conditions (Fig. $3 \mathrm{c}$ and Supplementary Fig. 2a). Moreover, expression of $R L M 1$, a transcription factor downstream of $\mathrm{Mkc1}$, was upregulated in the cas $5 \Delta /$ cas $5 \Delta$ mutant under basal conditions (Fig. 3d), suggesting strains lacking Cas5 experience intrinsic cell wall stress. Our results indicate Cas5 maintains cell wall homeostasis under basal conditions and during cell wall stress.

Cas5 is a transcriptional regulator of cell cycle dynamics. We observed an intriguing role of Cas5 on governing RNA PolII binding at genes important for cell cycle, meiosis, and DNA replication (Figs. 1c and 2c), suggesting Cas5 regulates cell cycle dynamics under basal and cell wall stress conditions. If this were the case, the expression pattern of cell cycle regulated genes would be perturbed in the absence of Cas5. Using the Candida cell cycle database $\mathrm{e}^{33}$, we determined that 56 out of the 604 genes that had Cas5-dependent RNA PolII binding under basal conditions, were genes whose expression peaked during the cell cycle (Supplementary Data 6). Of these, over 75\% (43 out of 56) had reduced RNA PolII binding in the cas $5 \Delta / \operatorname{cas} 5 \Delta$ mutant as compared with the wild-type strain (Fig. 4a and Supplementary Data 6). Next, we analyzed the class of genes involved in cell cycle that had reduced RNA PolII binding upon caspofungin treatment (Fig. 1e). Among these genes were all six factors of the minichromosome maintenance (MCM) complex (Fig. 4b), which has important roles in the formation of the pre-replicative complex (pre-RC) prior to DNA replication ${ }^{34}$. Reduced RNA PolII binding at genes encoding MCM complex components was dependent on Cas5 (Fig. 4b). As expected, qRT-PCR confirmed that reduced expression of representative genes, MCM2 and MCM3, was also dependent on Cas5 (Fig. 4c). Finally, we classified genes that were differentially bound by RNA PolII in response to caspofungin based on their peak expression throughout the cell cycle, once again using the Candida cell cycle database ${ }^{33}$. In the wild-type strain, genes with increased RNA PolII binding in response to caspofungin were predominantly G1-specific, and those with reduced RNA PolII binding were predominantly $\mathrm{M}$ phase-specific

Fig. 2 Cas5 regulatory network is rewired in response to caspofungin treatment. a, b Heat maps comparing Cas5-dependent genes with caspofunginregulated genes. a The left heat map shows the overlap of genes with Cas5-dependent increased RNA Polll binding with (+) and without (-) caspofungin treatment. Cas5-dependent genes are indicated by a solid blue line. The right heat map indicates those genes with increased binding (red) and decreased binding (blue) in a wild-type strain upon caspofungin treatment. b The left heat map shows the overlap of genes with Cas5-dependent reduced RNA Polll binding with $(+)$ and without $(-)$ caspofungin treatment. Cas5-dependent genes are indicated by a solid red line. The right heat map indicates those genes with increased (red) or decreased (blue) RNA Polll binding in a wild-type strain upon caspofungin treatment. c Bar graph showing the relative percentage of Cas5-dependent genes specific to basal conditions (blue bars), specific to caspofungin treatment (red bars), or common to both conditions (white bars) in the indicated KEGG pathways. The total number of genes belonging to each KEGG pathway are indicated. Only selected KEGG pathways are shown based on number of genes enriched for Polll binding either under basal or caspofungin conditions. See Supplementary Data 4 for complete data set. d Venn diagram depicting number of genes differentially bound in a cas $5 \Delta$ /cas $5 \Delta$ mutant relative to wildtype under basal conditions (increased, red; decreased, blue) and genes responding to caspofungin treatment in a wild-type strain (increased, green; decreased, yellow). The 131 genes highlighted in red text represent the substantial fraction of the genes with Cas5-dependent increase in RNA Polll binding under basal conditions that have reduced Polll binding in response to caspofungin 
a

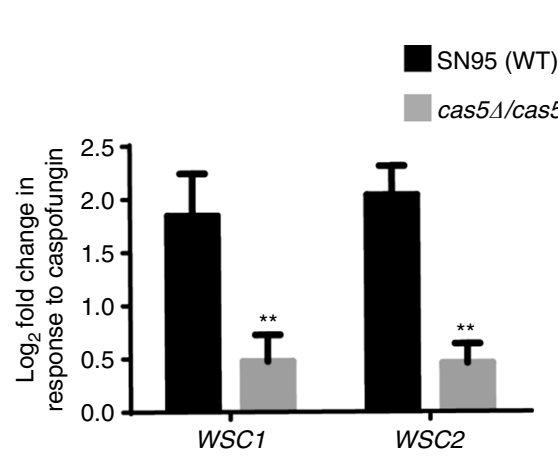

C

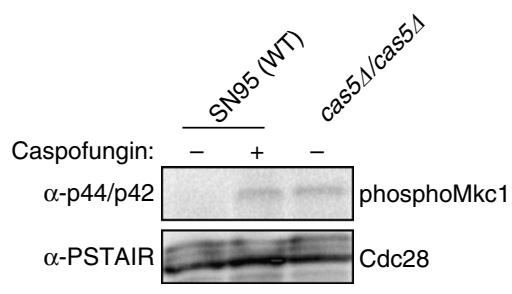

b

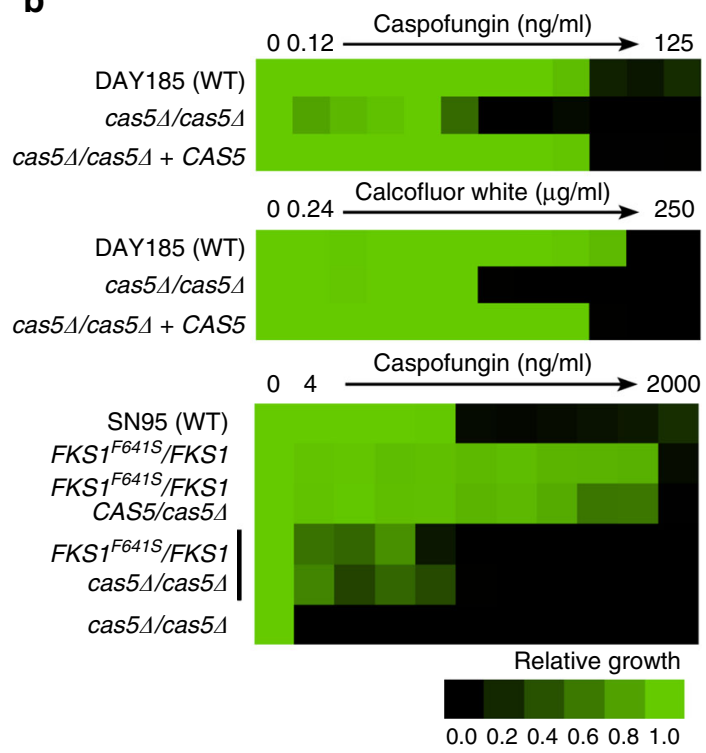

d

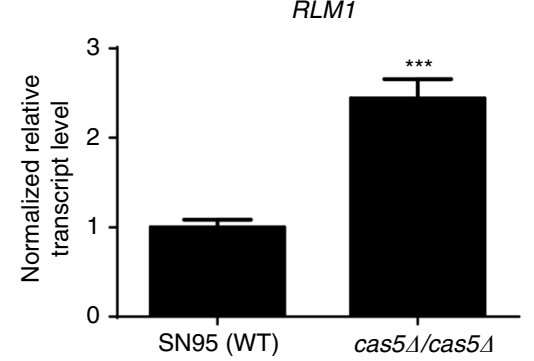

Fig. 3 Cas 5 regulates cell wall stability under basal and cell wall stress conditions. a WSC1 and WSC2, transmembrane sensors that respond to cell wall perturbation, require Cas5 for upregulation in response to caspofungin. The transcript level of WSC1 and WSC2 were monitored by qRT-PCR and normalized to TEF1. Plotted are the $\log _{2}$ fold-changes in transcript levels upon caspofungin treatment relative to untreated conditions in both the wildtype (black) and cas5 $\Delta /$ cas5 $\Delta$ mutant (gray). Error bars represent standard deviation (s.d.) from the mean of triplicate samples. Significance was measured with an unpaired $t$ test in GraphPad Prism ( $\left.{ }^{\star \star} P<0.01\right)$. b Cas5 is required for tolerance and resistance to cell wall stress. Caspofungin or calcofluor white susceptibility assays were conducted in YPD medium. Growth was measured by absorbance at $600 \mathrm{~nm}$ after $48 \mathrm{~h}$ at $30^{\circ} \mathrm{C}$. Optical densities were averaged for duplicate measurements. Data was quantitatively displayed with color using Treeview (see color bar). c Deletion of CAS5 leads to the activation of the cell wall integrity pathway in the absence of cell wall stress. A SN95 wild-type strain and a cas $5 \Delta / \operatorname{cas} 5 \Delta$ mutant were left untreated ( - ) or treated for $1 \mathrm{~h}$ with $125 \mathrm{ng} / \mathrm{ml}$ of caspofungin (+), as indicated. Phosphorylated Mkc1 was monitored by western blot and detected with $\alpha$-p44/42 antibody. Full blots are shown in Supplementary Fig. 2a. Cdc28 was detected with $\alpha$-PSTAIRE antibody and used as a loading control. $\mathbf{d}$ RLM1, a transcription factor downstream of $M k c 1$ in the cell wall integrity pathway, is induced in a cas $5 \Delta /$ cas $5 \Delta$ mutant in the absence of cell wall tress. The transcript level of RLM1 was monitored by qRT-PCR and normalized to TEF1. Differences in transcript level upon caspofungin treatment relative to untreated conditions are plotted. Error bars represent s.d. from the mean of triplicate samples. Significance was measured with an unpaired $t$ test in GraphPad Prism ( $\left.{ }^{\star \star \star} P<0.001\right)$

(Fig. 4d and Supplementary Data 6). Importantly, these trends were less apparent in a mutant lacking Cas5 (Fig. 4d and Supplementary Data 6). Overall, our results highlight the important role of Cas 5 in ensuring appropriate cell cycle dynamics under basal conditions and in response to cell wall stress.

Cas5 is activated by dephosphorylation. Given our findings that Cas5 governs cell cycle dynamics and cell wall stress responses, we next explored the mechanisms by which it is regulated. Initially, we monitored the subcellular localization of Cas 5 by indirect immunofluorescence in a strain in which both alleles of CAS5 were HA-tagged. The Cas5-HA protein was functional and sufficient to confer wild-type tolerance to caspofungin (Supplementary Fig. 3a). Under basal conditions, Cas5 appeared diffuse throughout the cytoplasm, however following treatment with caspofungin, Cas5 translocated to the nucleus (Fig. 5a). Owing to the moderate expression of Cas5 under its native promoter, we employed a strain in which we could overexpress Cas5 to facilitate its localization in the absence of stress. Overexpression of HA-tagged Cas5 confirmed that Cas5 was mostly cytoplasmic, as observed when under its native promoter (Fig. 5b). Overexpression also enabled us to observe a subpopulation of cells in which Cas5 was localized to the nucleus even in the absence of cell wall stress (Fig. 5b). Next, we assessed changes in Cas5 abundance in response to caspofungin. CAS5 transcript and protein levels were induced in response to cell wall stress, as measured by qRT-PCR and western blot analysis (Fig. 5c, d and Supplementary Fig. 2b). Thus, in response to cell wall perturbation by caspofungin, Cas 5 is induced and translocates to the nucleus.

When monitoring Cas5 protein levels, we observed that the Cas5 protein demonstrated a downward band shift when cells were exposed to cell wall antagonists, indicative of a posttranslational modification (Fig. 5e and Supplementary Fig. 2c). To confirm this shift was a consequence of cell wall stress, 
a

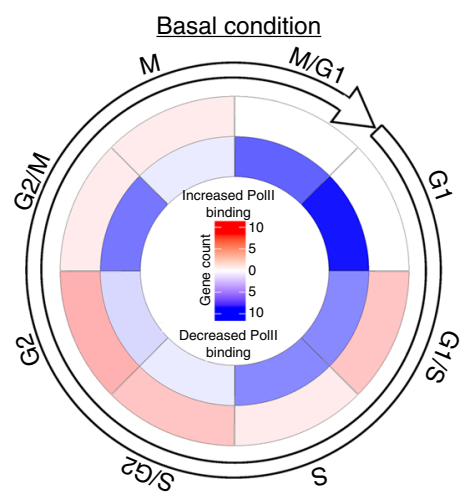

b

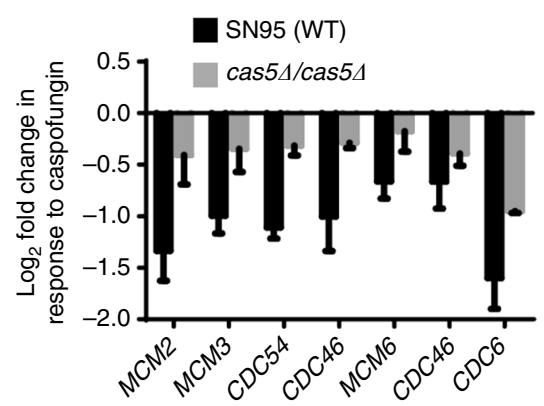

C
SN95 (WT)

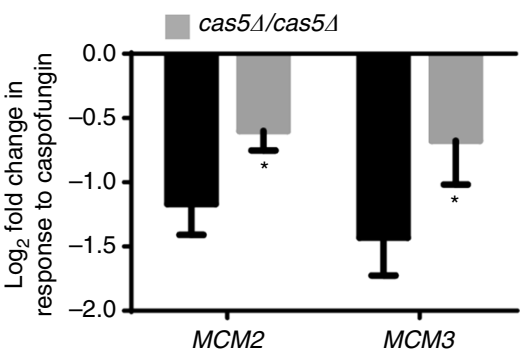

d

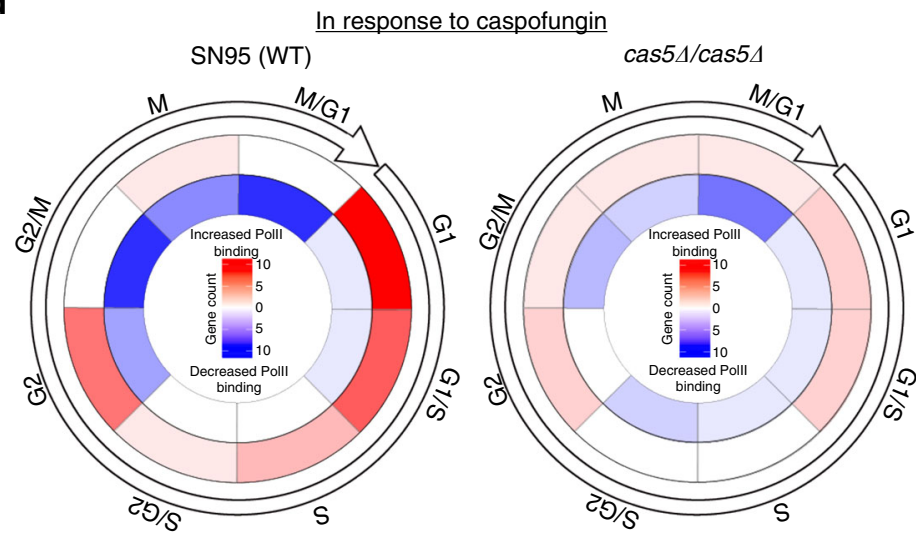

Fig. 4 Cas 5 governs cell cycle dynamics under basal and cell wall stress conditions. a Circos plot depicting the number of genes with increased (red) and decreased (blue) RNA Polll binding in the cas $5 \Delta / \operatorname{cas} 5 \Delta$ mutant whose normal expression in the wild-type peaks at the indicated cell cycle stage. $\mathbf{b}$ Cas 5 is required for the decreased Polll binding at genes encoding MCM complex members and CDC6 in response to caspofungin. Histogram showing the PollI ChIP-Seq signal for the indicated genes encoding MCM complex components in response to caspofungin treatment. Error bars represent standard deviation (s.d.) from the mean of duplicate samples. c Downregulation of MCM2 and MCM3 in response to caspofungin depends on Cas5. Transcript levels were monitored by qRT-PCR and normalized to TEF1. Error bars represent s.d. from the mean of triplicate samples. Significance was measured with an unpaired $t$ test in GraphPad Prism ( ${ }^{\star} P<0.05$ ). d Circos plots illustrating the number of genes with increased (red) or decreased (blue) Polll binding in response to caspofungin (CF) in wild-type and $\operatorname{cas} 5 \Delta / \operatorname{cas} 5 \Delta$ strains for genes whose expression is regulated throughout the cell cycle

we employed a strain in which we could specifically inhibit the kinase activity of the cell wall integrity regulator $\mathrm{Pkcl}^{35}$. In this strain, the only allele of PKC1 carries an M850G gatekeeper mutation, rendering the kinase susceptible to inhibition by ATP analog 1-naphthyl-PP1 (1-NA-PP1), without affecting its kinase activity in the absence of the inhibitor ${ }^{35}, 36$. In the presence of 1-NA-PP1, the gatekeeper mutant was hypersensitive to caspofungin, similar to a $p k c 1 \Delta / p k c 1 \Delta$ mutant (Supplementary Fig. 3b). Inhibition of Pkcl kinase activity induced Cas5 protein levels and resulted in a downward mobility shift (Fig. $5 \mathrm{f}$ and Supplementary Fig. 2d), similar to what was observed in a wild-type strain treated with caspofungin (Fig. 5d). Given that a change in protein phosphorylation is a common mechanism to regulate transcription factor activity ${ }^{37}$, we assessed whether Cas 5 was modified by phosphorylation. We performed two-dimensional gel electrophoresis coupled to western blot analysis. In the absence of stress, Cas5-HA resolved into a heterogeneous population of differentially charged species (Fig. $5 \mathrm{~g}$ ). In response to caspofungin, Cas5 collapsed into a less electronegative species, consistent with protein dephosphorylation. To confirm that Cas5 was phosphorylated under basal conditions, we monitored Cas5 mobility by western blot analysis following lambda phosphatase treatment. Phosphatase treatment led to a faster migrating Cas5 band, consistent with Cas5 being a phosphoprotein (Fig. 5h and 
Supplementary Fig. 2e). Finally, to determine if dephosphorylation of Cas5 is coupled to the activation of gene expression, we monitored transcript levels of ECM331 and PGA13, two Cas5-dependent caspofungin-responsive cell wall genes
(Supplementary Data 4) ${ }^{22}$. ECM331 and PGA13 were upregulated in wild-type cells with the same caspofungin exposure required to induce Cas5 dephosphorylation, and this upregulation was partially blocked by deletion of CAS5 (Fig. 5i). Thus, Cas5 is a

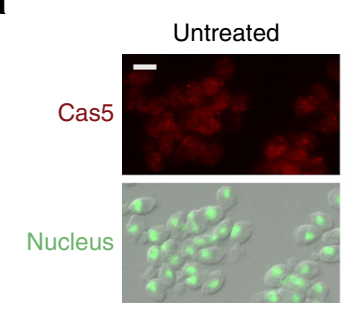

C

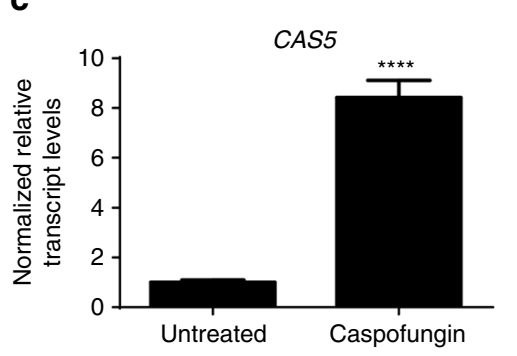

$\mathbf{f}$

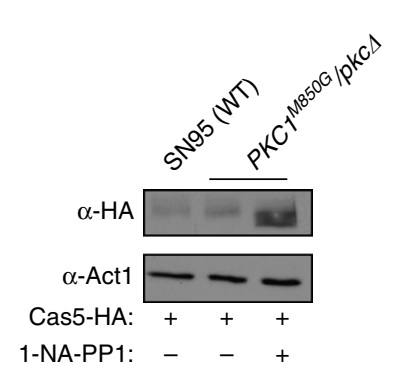

g d

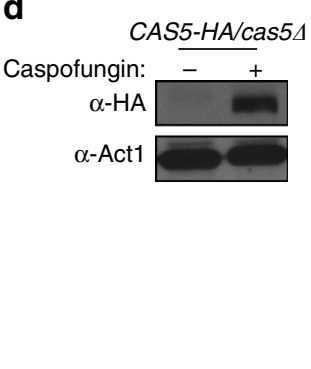

b
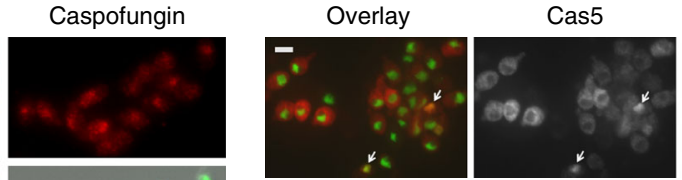

e

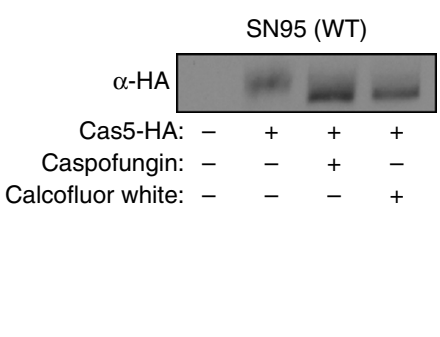

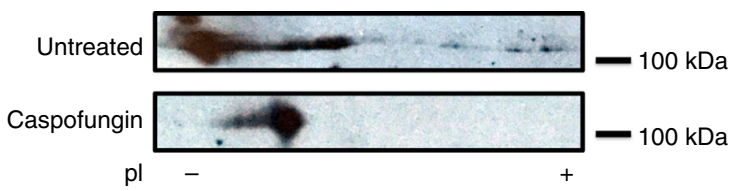

h

i

ECM331

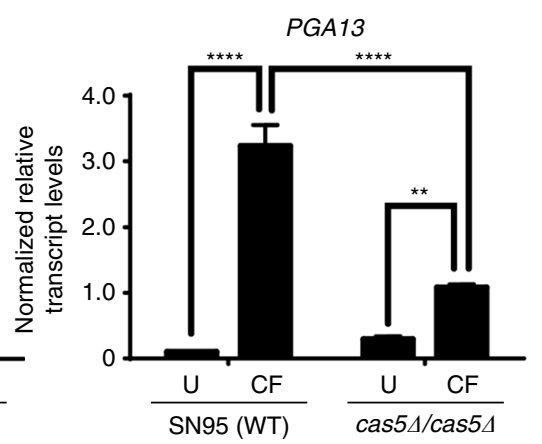

Fig. 5 Cas 5 becomes activated by dephosphorylation in response to cell wall stress. a Cas 5 localizes to the nucleus in response to caspofungin. Cells were fixed and Cas5 (red) was detected by indirect immunofluorescence using $\alpha$-HA antibody and $\alpha$-mouse IgG-Cy3. Nuclei (green) were visualized by DAPI staining. Scale bar represents $5 \mu \mathrm{m}$. b Cells were subcultured in rich medium to log phase and fixed. HA-tagged Cas 5 (red) and nuclei (green) were visualized as in a. Scale bar represents $5 \mu \mathrm{m}$. c CAS5 is induced by caspofungin. The transcript level of CAS5 was monitored by qRT-PCR and normalized to GPD1. Error bars represent standard deviation (s.d.) from the mean of triplicate samples. Significance was measured with an unpaired test in GraphPad Prism ( $\left.{ }^{\star \star \star \star} P<0.0001\right)$. d Levels of Cas 5 were monitored by western blot and detected with an $\alpha-\mathrm{HA}$ antibody. Actin was detected with an $\alpha-\beta$-actin antibody as a loading control. Full blots are shown in Supplementary Fig. 2 b. e Cas 5 is post-translationally modified upon cell wall stress treatment. Cells were grown to log phase and subsequently treated with $125 \mathrm{ng} / \mathrm{ml}$ of caspofungin or $50 \mu \mathrm{g} / \mathrm{ml}$ of calcofluor white for $1 \mathrm{~h}$. Total protein was resolved by SDS-PAGE and the blot was hybridized with an $\alpha-\mathrm{HA}$ to monitor Cas 5 migration. Full blots are shown in Supplementary Fig. $2 \mathrm{c}$. $\mathbf{f}$ Cas 5 migration and actin detection were monitored as part $\mathbf{d}$. Full blots are shown in Supplementary Fig. 2d. $\mathbf{g}$ Protein lysates were subjected to two-dimensional gel electrophoresis coupled with western blotting. Cas 5 was detected with an $\alpha-\mathrm{HA}$ antibody. $\mathbf{h}$ Cas 5 is phosphorylated in the absence of stress. Cas 5 migration was monitored by western blot and detected with an $\alpha-\mathrm{HA}$ antibody. Treatment of protein lysate with lambda phosphatase resulted in a faster migrating band. Full blots are shown in Supplementary Fig. 2e. i Cas5 is required for caspofungin (CF)-induction of cell wall genes. Transcript levels of ECM331 and PGA13 were monitored by qRT-PCR and normalized to GPD1. Error bars represent s.d. from the mean of triplicate samples. Significance was measured with a Tukey's multiple comparisons test in GraphPad Prism $\left({ }^{\star \star \star \star} P<0.0001,{ }^{\star \star} P<0.01\right)$ 
regulated by dephosphorylation and governs the expression of caspofungin-responsive cell wall genes in response to cell wall stress.

A Cas5 serine residue is critical for caspofungin tolerance. On the basis of our observations that Cas5 function is regulated by phosphorylation, we hypothesized that dephosphorylation of key residues is important for Cas 5 activation. To map the phosphorylation residues on Cas5 important for its activity, we performed immunoprecipitation coupled to mass spectrometry with HA-tagged Cas5. Phosphorylation was detected at serine residues S462 and S476 (Supplementary Fig. 4a). To test whether these serine residues along with two other adjacent residues, S464 and S472, were functionally important for Cas5 function in regulating cell wall integrity, we mutagenized all four serines to either glutamic acid, to mimic a constitutively phosphorylated state, or alanine, to mimic a constitutively unphosphorylated state. The resulting mutants displayed wild-type tolerance to caspofungin (Supplementary Fig. 4b), with no appreciable difference in the band shift upon caspofungin treatment (Supplementary Fig. 4c), suggesting that the sites identified by mass spectrometry were not sufficient to control Cas5 function.

To identify other potential Cas5 phosphorylation sites, we analyzed its amino acid sequence using NetPhos and followed up on S769, a highly conserved residue in one of the zinc finger domains with a phosphorylation site-prediction score of 0.980 (Fig. 6a $)^{38,39}$. To assess the functional significance of this residue, we engineered strains in which one allele of CAS5 was deleted and the other was replaced with a wild-type allele, the phosphomimetic S769E allele, or the phosphodeficient S769A allele. Introduction of the phosphomimetic S769E allele as the only source of CAS5 phenocopied homozygous deletion of CAS5 in terms of hypersensitivity to caspofungin, whereas introduction of the phosphodeficient S769A allele conferred a wild-type phenotype (Fig. 6b). Consistent with our hypothesis, the S769E mutation blocked the upregulation of ECM331 and PGA13 in response to caspofungin, whereas the S769A mutation did not (Fig. 6c). Notably, these mutations did not affect Cas5 induction or dephosphorylation in response to caspofungin (Fig. $6 \mathrm{~d}$ and Supplementary Fig. 2f), suggesting that the S769E substitution does not affect the capacity of Cas5 to sense cell wall stress, and that additional phosphorylation sites remain to be identified. Our data supports a model in which Cas5 is activated by dephosphorylation in response to cell wall stress.

Glc7 dephosphorylates Cas5 in response to cell wall stress. To identify the phosphatase that regulates Cas 5 activity, we leveraged insights from the model yeast S. cerevisiae. A BLASTp search of the $C$. albicans Cas5 protein sequence against the $S$. cerevisiae protein database identified Mig1, Msn4, Mig2, and Msn2 as proteins with similar sequences ${ }^{40}$. Mig1, Msn4, and Msn2 are dephosphorylated by the type I protein phosphatase Glc $7^{41,42}$. As Glc7 influences cell wall maintenance in $S$. cerevisiae ${ }^{43}$, we hypothesized that Glc7 was required for activating cell wall stress responses in C. albicans. We engineered a strain in which we could transcriptionally repress GLC7 by deleting one allele and replacing the native promoter of the remaining allele with a tetracycline-repressible promoter. We confirmed that GLC7 expression was repressed in the presence of the tetracycline analog doxycycline by qRT-PCR (Supplementary Fig. 5a). Depletion of $G L C 7$ with doxycycline conferred hypersensitivity to caspofungin (Fig. 7a), confirming Glc7 is important for tolerating cell wall stress in C. albicans. Next, we examined the effect of GLC7 depletion on Cas5 expression and function. Doxycyclinemediated transcriptional repression of GLC7 did not block upregulation of Cas5 in response to caspofungin (Fig. $7 \mathrm{~b}$ and Supplementary Fig. 2g), but did block the downward band shift associated with dephosphorylation, as was evident with equivalent Cas5 levels loaded per sample (Fig. 7c and Supplementary Fig. 2h). Depletion of GLC7 also blocked the upregulation of Cas5-dependent caspofungin-responsive genes, ECM331 and PGA13 (Fig. 7d). Thus, Glc7 activates Cas5 by dephosphorylation in response to cell wall stress, thereby enabling transcriptional upregulation of cell wall genes.

Cas5 governs cell wall stress responses with Swi4/Swi6. To identify physical interactors of Cas5, we leveraged the results from our immunoprecipitation coupled to mass spectrometry analysis (Supplementary Data 7). The only interaction that passed a stringent statistical analysis using Significance Analysis of Interactome (SAINT) ${ }^{44}$ with a probability threshold of 0.9 was Swi4 (Supplementary Data 7), which interacts with Swi6 to form the SBF complex in S. cerevisiae ${ }^{45}$. To validate the interaction between Cas5 and the SBF complex, we individually tandem affinity purification (TAP) tagged Swi4 or Swi6 at the C terminus in a strain harboring Cas5-HA to enable co-immunoprecipitation analysis. The Swi4-TAP and Swi6-TAP proteins were functional and sufficient to mediate wild-type tolerance to caspofungin (Supplementary Fig. 5b). Immunoprecipitation of Cas5 with anti-HA agarose co-purified both Swi4 and Swi6 (Fig. 8a and Supplementary Fig. 6), verifying these proteins physically interact. To determine whether the SBF complex has a role in cell wall stress responses in C. albicans, we monitored the expression of ECM331 and PGA13 in mutants lacking Swi4, Swi6, or Cas5. In all three mutants, upregulation of these cell wall genes in response to caspofungin was blocked (Fig. 8b). However, strains lacking Swi4 or Swi6 displayed intermediate caspofungin susceptibility phenotypes relative to a wild-type strain and a CAS5 homozygous deletion mutant (Fig. 8c). Altogether, our results suggest that Cas5 regulates transcriptional responses to cell wall stress in part through the SBF complex and in part through an independent mechanism.

Cas5 governs cell cycle dynamics independent of Swi4/Swi6. Since our RNA PolII ChIP-Seq data implicated Cas5 in proper cell cycle dynamics and DNA replication (Figs. 1c and 4), we wanted to further investigate the impact of Cas5 on nuclear division. We monitored the number of nuclei per cell in wildtype and $\operatorname{cas} 5 \Delta / \operatorname{cas} 5 \Delta$ strains. Cells were stained with DAPI for DNA content and calcofluor white for chitin (Fig. 9a and Supplementary Fig. 7a). During log phase, $\sim 40 \%$ of cells lacking Cas5 were multinucleated, an indication of uncontrolled DNA replication or a defect in cytokinesis (Fig. 9b). Given that Swi4 and Swi6 physically interact with Cas5 (Fig. 8a) and that the SBF complex regulates the G1/S phase progression in S. cerevisiae and C. albicans $^{46,47}$, we also investigated their impact on nuclear division. Strikingly, $<10 \%$ of mutant cells lacking either Swi4 or Swi6 showed a multinucleated phenotype, suggesting that the SBF complex has a minor role in ensuring proper nuclear division in C. albicans (Fig. 9a, b). We further investigated the defective nuclear division associated with loss of Cas5 by time-lapsed fluorescence microscopy. To monitor DNA content, we RFPtagged histone H4, encoded by HHF1; to monitor mitotic spindle positioning, we GFP-tagged the Dam1 complex subunit DAD2 (Fig. 9c, d). We observed multiple nuclei dividing simultaneously in a single cell in a cas5 $5 /$ cas5 $\Delta$ mutant (Fig. 9d and Supplementary Fig. 7b). Importantly, each nucleus had at least one spindle pole body, suggesting that the accumulation of nuclei was due to nuclear division and not spontaneous nuclear fragmentation (Fig. 9d and Supplementary Fig. 7b). 
a

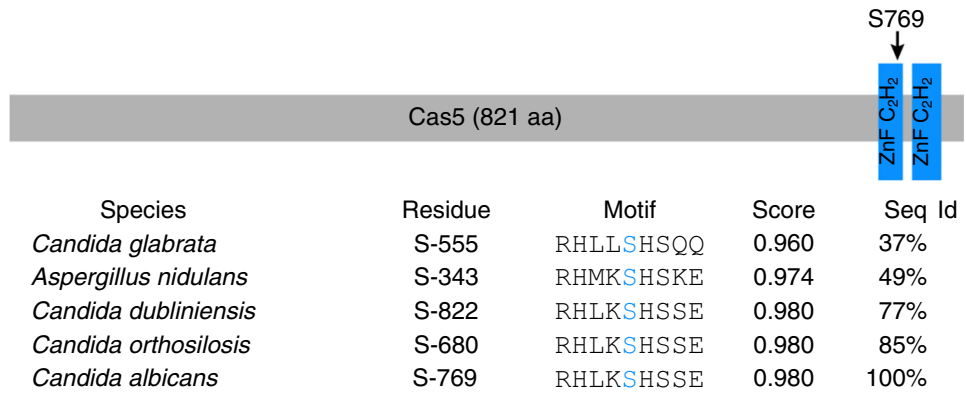

b
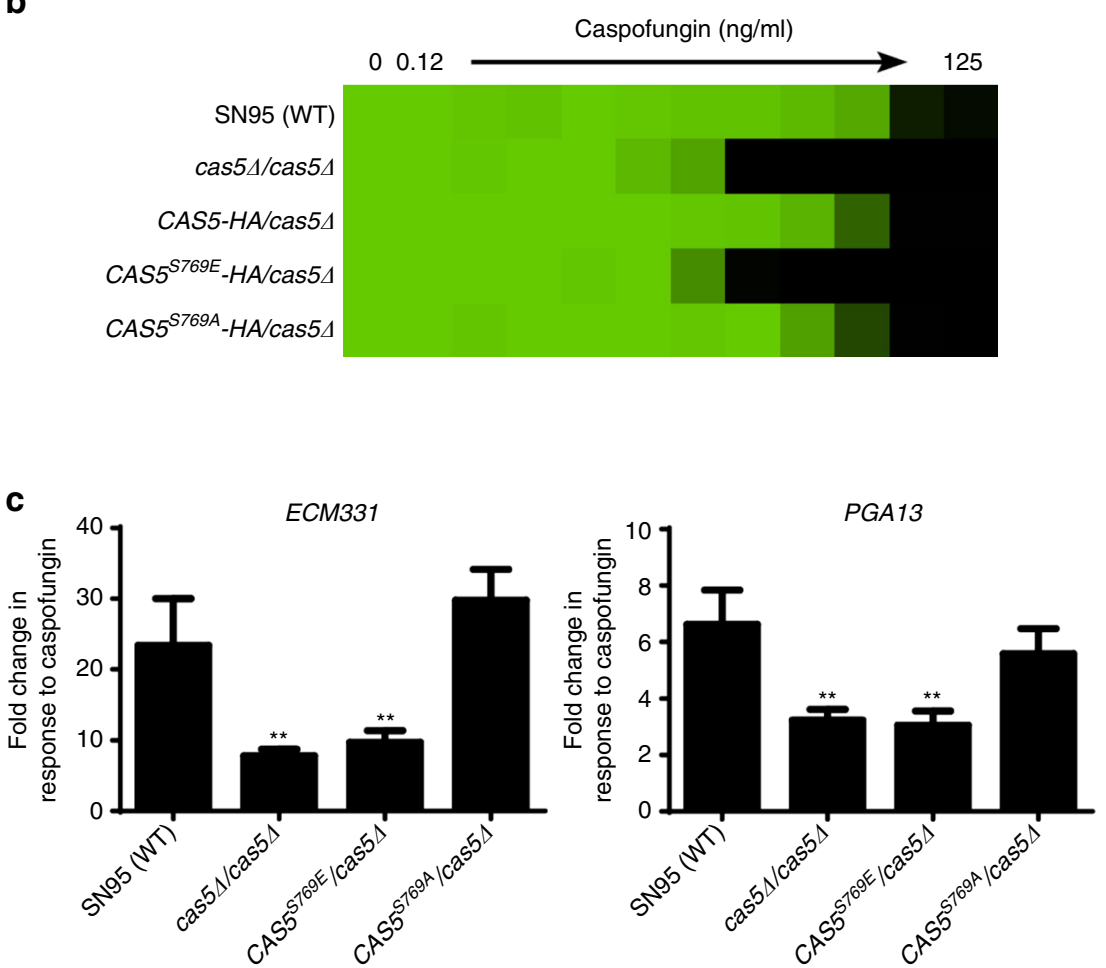

d

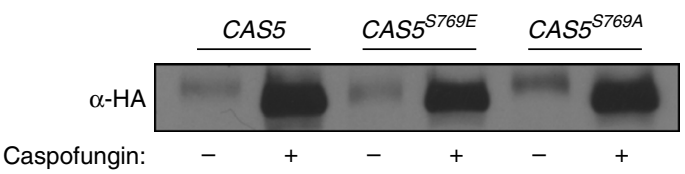

Fig. 6 The S769E substitution in the Cas5 DNA-binding domain phenocopies CAS5 deletion. a Schematic showing the position of S769 in the Cas5 zinc finger domain and the alignment of Cas 5 orthologs in related fungal species. The alignment was performed using the Candida Genome Database (CGD), and the phosphorylation site-prediction score was generated using NetPhos. $\mathbf{b}$ The phosphomimetic S769E substitution in Cas 5 confers hypersensitivity to caspofungin. Caspofungin susceptibility assays were conducted in YPD medium. Growth was measured by absorbance at $600 \mathrm{~nm}$ after $48 \mathrm{~h}$ at $30{ }^{\circ} \mathrm{C}$. Optical densities were averaged for duplicate measurements. Data was quantitatively displayed with color using Treeview (see color bar in Fig. 2). c The S769E substitution blocks induction of cell wall genes in response to caspofungin treatment. The transcript levels of ECM 331 and PGA13 were monitored by qRT-PCR and normalized to GPD1. Plotted are the fold-changes in transcript levels of ECM331 or PGA13 in response to caspofungin relative to untreated. Error bars represent standard deviation (s.d.) from the mean of triplicate samples. The fold change in gene expression for each mutant strain was compared with the wild-type strain using one-way ANOVA in GraphPad Prism ( $\left.{ }^{\star \star} P<0.01\right)$. d Phosphomutations in CAS5 do not affect band shifts associated with activation of the cell wall stress response, as observed upon caspofungin treatment. Cas 5 was monitored by western blot and detected using an $\alpha-\mathrm{HA}$ antibody. Full blots are shown in Supplementary Fig. $2 f$

Next, we assessed the ploidy of the cas $5 \Delta / \operatorname{cas} 5 \Delta$ mutant compared with a wild-type control by flow cytometry. During log phase growth in the absence of external stress, wild-type cells exhibited two distinct peaks at $2 \mathrm{C}$ and $4 \mathrm{C}$ (representing the G1- and G2-phases of the cell cycle, respectively), whereas the cas $5 \Delta /$ cas $5 \Delta$ mutant exhibited five distinct peaks at $2 \mathrm{C}, 4 \mathrm{C}, 6 \mathrm{C}$, 8C, and $12 \mathrm{C} \mathrm{(Fig.} \mathrm{9e} \mathrm{and} \mathrm{Supplementary} \mathrm{Fig.} \mathrm{7c).} \mathrm{Notably,} \mathrm{peaks}$ at $1 \mathrm{C}$ and $3 \mathrm{C}$ were absent, indicating that the nuclei were not haploidizing, mating, or undergoing reductional division. Instead, these data suggest multiple rounds of DNA replication were 
a

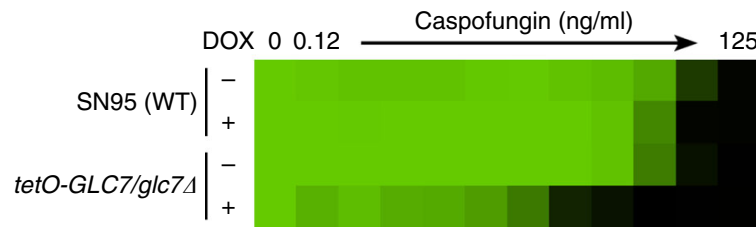

b

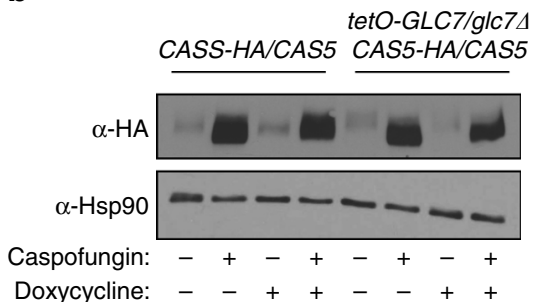

C

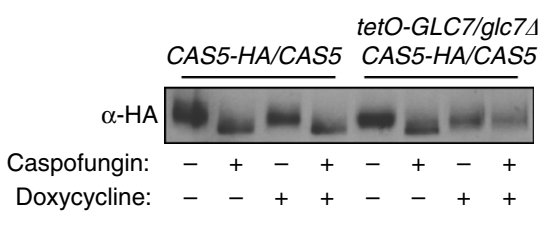

d
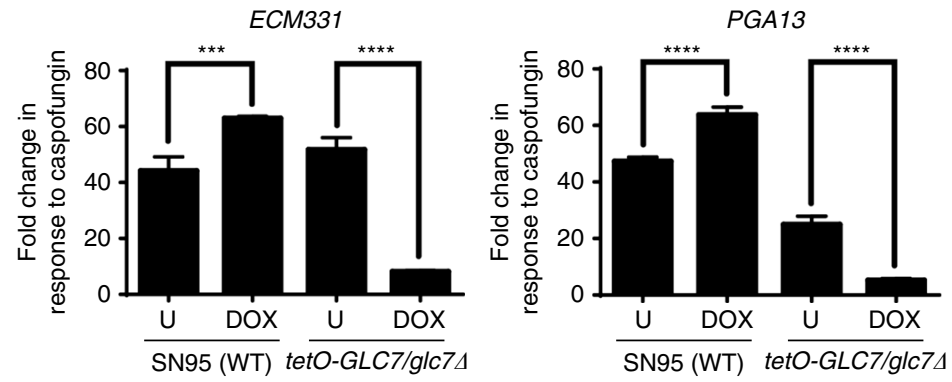

Fig. 7 Cas 5 activation in response to caspofungin is coupled with dephosphorylation by the protein phosphatase Glc7. a Depletion of GLC7 confers sensitivity to caspofungin. Caspofungin susceptibility assays were conducted in YPD medium in the presence or absence of $1 \mu \mathrm{g} / \mathrm{ml}$ of doxycycline (DOX). Growth was measured by absorbance at $600 \mathrm{~nm}$ after $48 \mathrm{~h}$ at $30^{\circ} \mathrm{C}$. Optical densities were averaged for duplicate measurements. Data were quantitatively displayed with color using Treeview (see color bar in Fig. 2). b Upregulation of Cas5 expression does not depend on Glc7. CAS5-HA/CAS5 and CAS5-HA/CAS5 tetO-GLC7/glc7D strains were cultured in the absence or presence of doxycycline and caspofungin, as indicated. Cas 5 was monitored by western blot and detected with an $\alpha-\mathrm{HA}$ antibody. Hsp90 protein levels served as a loading control. Full blots are shown in Supplementary Fig. $2 \mathrm{~g}$. c Post-translational modification of Cas 5 is absent upon GLC7 depletion. The western blot was performed as described in $\mathbf{b}$, except caspofungin treated samples were diluted fivefold to achieve equal loading of Cas5. Full blots are shown in Supplementary Fig. 2h. $\mathbf{d}$ Dephosphorylation of Cas 5 is required for the induction of cell wall genes in response to caspofungin treatment. CAS5-HA/CAS5 and CAS5-HA/CAS5 tetO-GLC7/g/c7D strains were grown in the absence or presence of doxycycline (DOX) or caspofungin, as indicated. The transcript levels of ECM331 and PGA13 were monitored by qRT-PCR and normalized to GPD1. Plotted are the fold-changes in transcript levels in the presence of caspofungin relative to untreated. Error bars represent standard deviation (s.d.) from the mean of triplicate samples. The treatment conditions were compared using a Tukey's multiple comparisons test in GraphPad Prism $\left({ }^{\star \star \star \star} P<0.0001,{ }^{\star \star \star} P<0.001\right)$

occurring in the same cell, causing ploidy level increases similar to what is observed when C. albicans is exposed to fluconazole ${ }^{48}$.

Finally, to determine whether the dephosphorylated form of Cas5 was required for controlled DNA replication, we quantified the number of nuclei per cell in the phosphomutants. The CAS5 mutant carrying the phosphomimetic S769E substitution phenocopied the cell cycle defects observed in the CAS5 homozygous deletion mutant, whereas the mutant carrying phosphodeficient S769A substitution was indistinguishable from the wildtype (Fig. 9f). Thus, our results support a novel role for the dephosphorylated form of Cas5 in regulating nuclear division that is largely independent of the SBF complex.

\section{Discussion}

The capacity to coordinate cell cycle progression with stress responses is crucial for cellular survival to give cells time to recover from a myriad of environmental perturbations, including cell wall stress ${ }^{49}$. In the present work, we uncovered a dual role for the transcription factor Cas5 in mediating cell cycle dynamics and cell wall stress responses in C. albicans (Fig. 10). We discovered that RNA PolII binding to many genes important for cell wall biosynthesis and DNA replication is dependent on Cas5 under basal conditions (Fig. 1). We also found Cas5 regulates the expression of a distinct set of cell wall and cell cycle genes in response to cell wall stress (Fig. 2). We established Cas5 function is regulated by the protein phosphatase Glc7 (Fig. 7), and Cas5 functions in concert with Swi4 and Swi6 to regulate cell wall homeostasis (Fig. 8). Our findings implicate Cas5 at the core of a novel mechanism by which cell cycle and cell wall integrity are coordinately regulated.

The coordination of sensing and responding to cell wall stress is crucial for fungi, and precise temporal control of cell wall stress response regulators underpins the rapid mobilization of signaling cascades that govern cellular integrity. We demonstrated that Cas 5 not only becomes activated in response to cell wall stress but it also maintains cell wall stability under basal conditions (Figs. 3 and 5). Although Cas5 is not broadly conserved in the fungal kingdom, its activation is regulated by a conserved protein phosphatase, Glc7, which has $91 \%$ protein identity to the S. cerevisiae ortholog. In S. cerevisiae, temperature sensitive glc7 mutants exhibit sorbitol-remediable lysis defects at the restrictive 
a

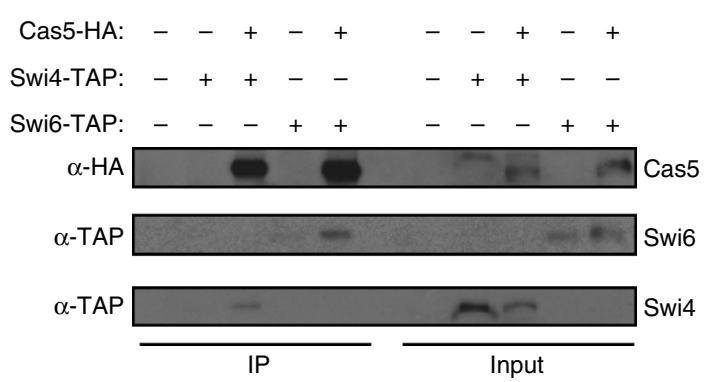

b

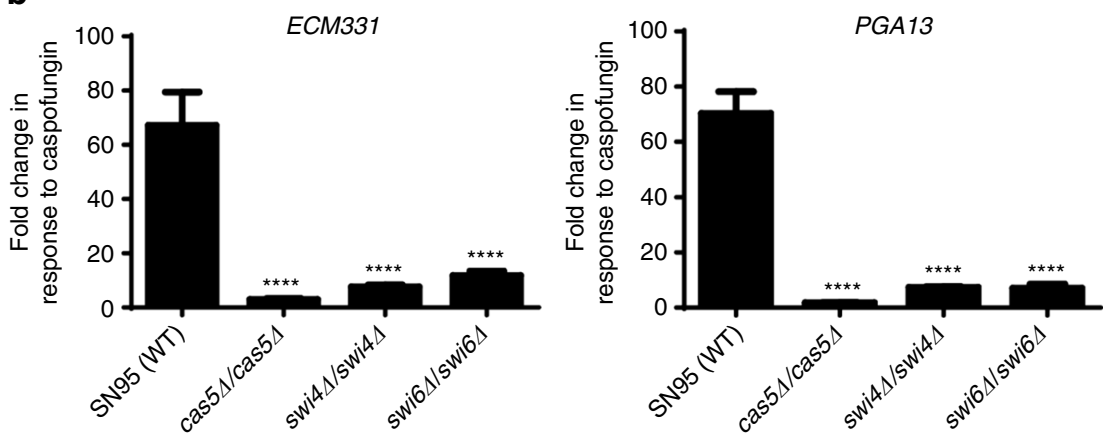

C

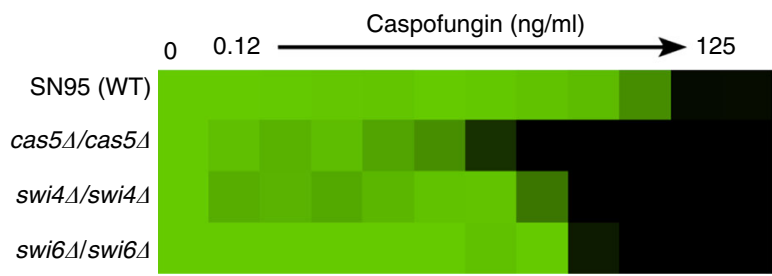

Fig. 8 Cas5 regulates caspofungin tolerance in part through the interaction with components of the SBF complex, Swi4 and Swi6. a Swi4 and Swi6 co-purify with Cas5. C terminally HA-tagged Cas 5 was immunoprecipitated with $\alpha$-HA beads. Swi 4 and Swi6 co-purification was monitored by western blot and detected with an $\alpha$-TAP antibody. Cas 5 pull down was confirmed by detection with an $\alpha-\mathrm{HA}$ antibody. Input samples confirm the expression of tagged proteins. Full blots are shown in Supplementary Fig. 6. b Cas5, Swi4, and Swi6 are required for the upregulation of caspofungin-responsive genes. The transcript levels of ECM331 and PGA13 was monitored by qRT-PCR and normalized to GPD1. Plotted are the fold-changes in transcript levels in the presence of caspofungin relative to untreated. Error bars represent standard deviation (s.d.) from the mean of triplicate samples. The fold change in gene expression for each mutant strain was compared to the wildtype using one-way ANOVA in GraphPad Prism ( ${ }^{\star \star \star \star} P<0.0001$ ). c Swi4 and Swi6 regulate cell wall stress response. Caspofungin susceptibility assays were conducted in YPD medium. Growth was measured by absorbance at $600 \mathrm{~nm}$ after $48 \mathrm{~h}$ at $30{ }^{\circ} \mathrm{C}$. Optical densities were averaged for duplicate measurements. Data was quantitatively displayed with color using Treeview (see color bar in Fig. 2)

temperature, due to impaired cell wall integrity ${ }^{43}$. We demonstrated Glc7 is required for cell wall maintenance in C. albicans (Fig. 7). Glc7 substrates in S. cerevisiae include three proteins with sequence similarity to Cas5: Mig1, Msn2, and Msn4. Msn2 and Msn4 are transcription factors that are required for general stress response in $S$. cerevisiae, but not in C. albicans ${ }^{50,51}$. This highlights a divergence in signal transduction cascades required to respond to environmental stressors, and suggests that Cas5 may be the central downstream effector of Glc7 that modulates stress responses in C. albicans. Consistent with this possibility, Cas5 controls responses not only to cell wall stress, but also to cell membrane stress exerted by the azole antifungal drugs ${ }^{52}$.

In yeast, cell wall stress originates not only through environmental perturbations but also through normal physiological changes that are coupled to cell cycle progression, as cell wall remodeling and biosynthesis are required to enable the emergence of the growing daughter bud. In S. cerevisiae cell membrane perturbation triggers cell cycle arrest via degradation of Cdc6, a component of the pre- $\mathrm{RC}^{53}$. This is required for cell survival as continued cell cycle progression in the presence of plasma membrane damage induces plasma membrane rupture and cell lysis, leading to cell death ${ }^{53}$. We found that CDC6 and genes encoding members of the MCM complex that participate in the pre-RC are transcriptionally repressed in response to caspofungin in a Cas5-dependent manner (Fig. 4). To our knowledge, this is the first mechanistic insight into the signaling events that couple cell wall stress with cell cycle arrest in C. albicans, and likely enables the cell to respond to environmental stress without succumbing to cell death. In S. cerevisiae, cell cycle genes are regulated by transcriptional complexes composed of Swi4 and Swi6, including the SBF complex, which is involved in budding and membrane/cell-wall biosynthesis ${ }^{54}$. We observed that impairment of Cas5 function manifested in more severe misregulation of nuclear division than deletion of SWI4 or SWI6 (Fig. 9), suggesting Cas5 regulates nuclear division largely independent of the SBF complex.

Targeting regulators of cellular circuitry that are crucial for cellular stress responses may provide a powerful strategy for antifungal drug development. This strategy opens up the opportunity for combining stress response inhibitors with current antifungals for treatment of fungal infections. Promising examples of this approach include targeting the molecular chaperone 
a
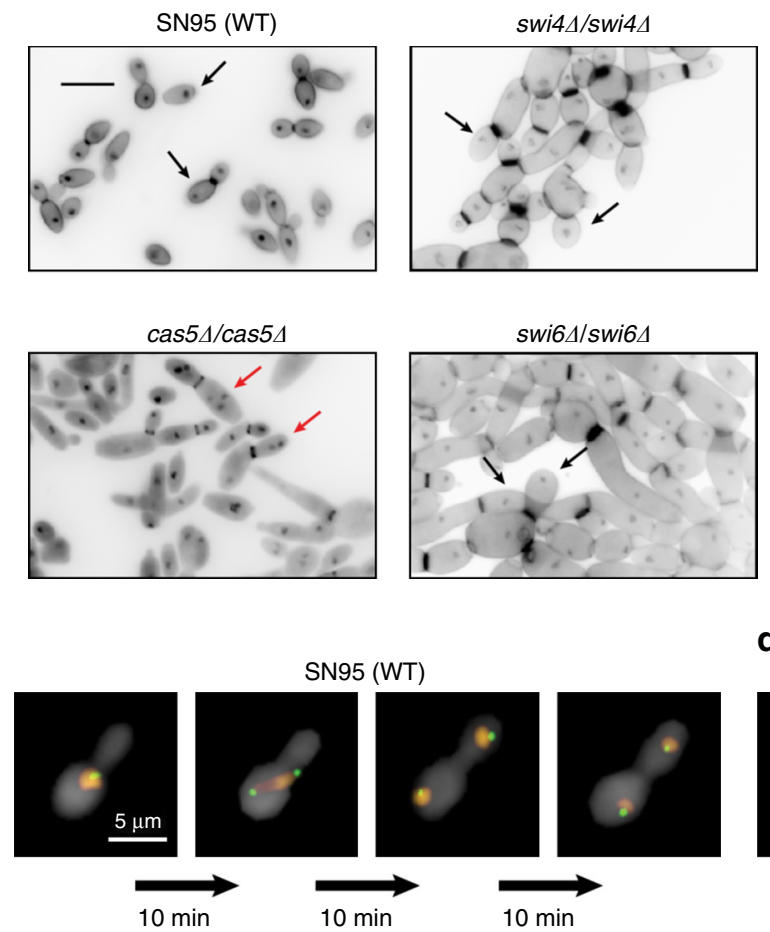

e
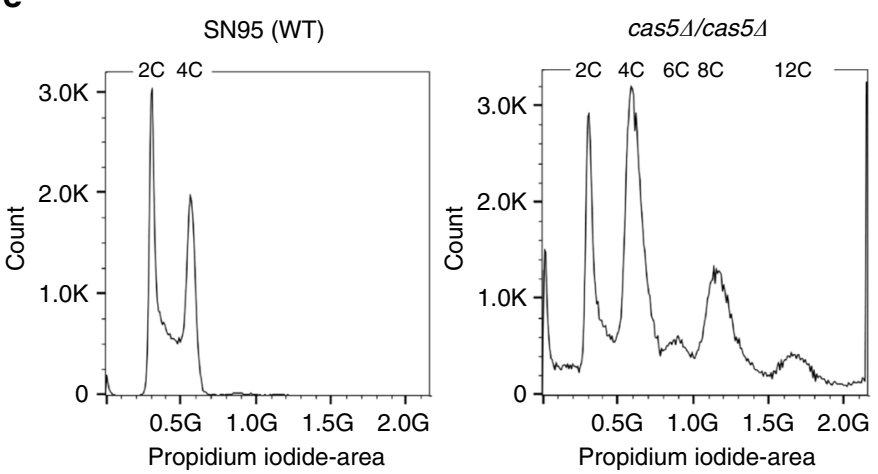

b

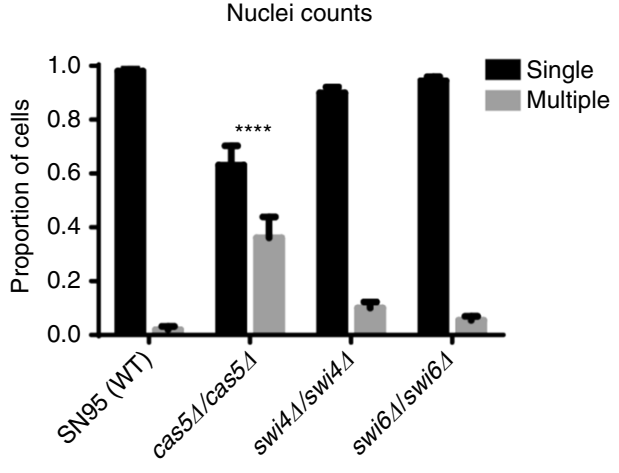

d

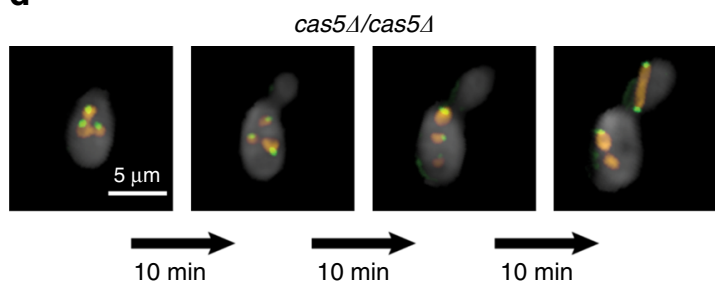

$\mathbf{f}$

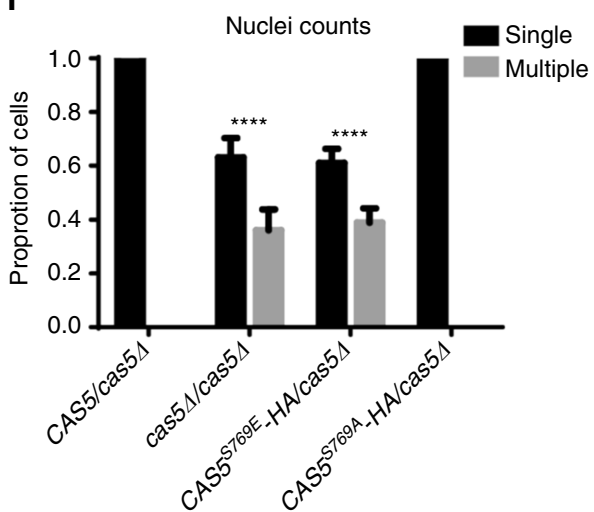

Fig. 9 Cas 5 regulates nuclear division largely independent of Swi4 and Swi6. a Nuclei were stained with DAPI and chitin was stained using calcofluor white. For each image, 12 Z-stack slices were taken at $0.3 \mu \mathrm{m}$ each. Black arrows highlight cells with a single nucleus. Red arrows highlight cells with a multinucleated phenotype. b Mutants lacking Cas 5 exhibit severe cell cycle defect. The histogram represents the average number of nuclei per cell in three biological replicates. The number of nuclei counted in each experiment was at least 120 cells for each strain. The nuclei counts for each strain were averaged and error bars represent standard deviation (s.d.) from the mean of biological duplicates. Nuclei counts for each strain were compared to the wildtype using one-way ANOVA in GraphPad Prism ( ${ }^{\star \star \star \star} P<0.0001$ ). c, d Mitotic spindles are aligned along the mother-bud axis during cell division in wild-type cells (c) and misaligned in a mutant lacking Cas5 (d). DNA was monitored by RFP-tagged Hhf1, and mitotic spindle was monitored by GFPtagged Dad2 using time-lapse fluorescence microscopy. e Cas5 is required for maintaining normal DNA content throughout the cell cycle. Cellular DNA content was measured by propidium iodide and flow cytometry of the wild-type diploid and the cas $5 \Delta / \operatorname{cas} 5 \Delta$ mutant strain. The wild-type diploid has the standard G1 and G2 cell cycle peaks representing $2 \mathrm{C}$ and $4 C$ DNA levels. The cas $5 \Delta / \operatorname{cas} 5 \Delta$ mutant population, in addition to the same $2 C$ and $4 C$ DNA levels, contained a large subpopulation of cells with DNA levels at $6 \mathrm{C}, 8 \mathrm{C}$, and $12 \mathrm{C}$. These DNA levels represent tetraploid ( $4 \mathrm{~N})$ and hexaploid (6N) cells. f Activation of Cas 5 by dephosphorylation is required for proper cell cycle progression. The histogram represents the average number of nuclei per cell in three biological replicates. The number of nuclei counted was at least 120 cells for each strain. The nuclei counts for each strain were averaged and error bars represent s.d. from the mean of biological duplicates. Nuclei counts for each strain were compared to that of the wildtype using one-way ANOVA in GraphPad Prism ( $\left.{ }^{\star \star \star \star} P<0.0001\right)$

Hsp90 or its downstream effector calcineurin, which are both required for drug resistance and virulence of diverse fungal pathogens ${ }^{8,55}$. Given that both of these regulators are highly conserved in humans and have essential functions in mobilizing immune responses, the challenge in exploiting the therapeutic utility of these targets for antifungal therapies hinges on the development of fungal-selective inhibitors ${ }^{56}$. Our work further supported the role of Cas5 in governing echinocandin tolerance and established that Cas5 enables echinocandin resistance in a strain harboring a mutation in the drug target gene FKS1 (Fig. 3b). As Cas5 lacks an identifiable ortholog in humans ${ }^{22}$, but is required for drug resistance (Fig. 3b) and virulence in C. albicans ${ }^{10,57}$, it provides an attractive target for antifungal drug development. There is growing support for the feasibility of 


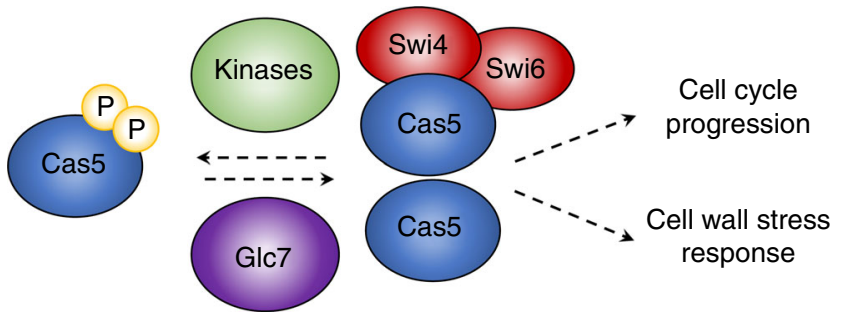

Fig. 10 Model for Cas5-mediated coupling of cell cycle progression to cell wall stress response. Cas 5 is dephosphorylated by the protein phosphatase Glc7 in response to cell wall stress. Once dephosphorylated, it can translocate to the nucleus to govern the expression of a myriad of genes, including those important for cell cycle regulation and cell wall stress response. Cas 5 regulates changes in gene expression through both Swi4/Swi6-dependent and independent mechanisms

chemical modulation of transcription factors based on blocking transcription factor dimerization, DNA binding, or the interaction with regulatory proteins ${ }^{58,59}$. Elucidating mechanisms that enable remodeling of transcriptional programs that control stress response and virulence traits provides opportunities to expand the antimicrobial target space in this era of antimicrobial resistance.

\section{Methods}

Strains and reagents. All C. albicans strains were archived in $25 \%$ glycerol and stored at $-80{ }^{\circ} \mathrm{C}$. Strains were grown in YPD (1\% yeast extract, $2 \%$ bactopeptone, and $2 \%$ glucose) at $30{ }^{\circ} \mathrm{C}$ unless otherwise specified. For solid media, $2 \%$ agar was added. Strains were constructed according to standard protocols. Strains used in this study are listed in Supplementary Table 1. Caspofungin was generously provided by Terry Roemer (Department of Infectious Diseases, Merck Research Laboratories) and was diluted to a $100 \mu \mathrm{g} / \mathrm{ml}$ stock, and used at a final concentration of $125 \mathrm{ng} / \mathrm{ml}$. Doxycycline (DOX, BD Biosciences \#631311) was formulated in a $20 \mathrm{mg} / \mathrm{ml}$ stock in water and used at a final concentration of $0.02 \mu \mathrm{g} / \mathrm{ml}$ or $1 \mu \mathrm{g} / \mathrm{ml}$, as specified.

Strain construction. CaLC2034: To generate a CAS5 heterozygous deletion mutant, the NAT flipper cassette (pLC49) ${ }^{60}$ was PCR amplified using primers oLC2017 and oLC2018 (4366 bp) and transformed into CaLC239 (SN95).

NAT-resistant transformants were PCR tested with oLC275 in combination with oLC2034 for upstream integration and oLC274 in combination with oLC2035 for downstream integration. The SAP2 promoter was induced to drive expression of FLP recombinase to excise the NAT flipper cassette.

CaLC2056: To generate a CAS5 homozygous deletion mutant, the NAT flipper cassette (pLC49) ${ }^{60}$ was PCR amplified as described for CaLC2034 and transformed into CaLC2034. NAT-resistant transformants were PCR tested for correct integration of the cassette as described for CaLC2034. The presence of deleted allele was verified by amplification with primer pairs oLC2034 in combination with oLC2035, and absence of wild-type allele was verified by amplification with primer pairs oLC2047 in combination with oLC2048. The SAP2 promoter was induced to drive expression of FLP recombinase to excise the NAT flipper cassette.

CaLC3908/CaLC3909: To generate a CAS5 heterozygous deletion mutant in a strain carrying the FKS1 ${ }^{F 641 S}$ mutation, the NAT flipper cassette (pLC49) ${ }^{60}$ was PCR amplified as described for CaLC2034 and transformed into CaLC2087 (SN95 FKS1 ${ }^{F 641 S}$ /FKS1). Correct upstream and downstream integration at the CAS5 locus was verified as described for CaLC2034. The SAP2 promoter was induced to drive expression of FLP recombinase to excise the NAT flipper cassette. To generate a CAS5 homozygous deletion mutant, the NAT flipper cassette (pLC49) ${ }^{60}$ was again PCR amplified and transformed into the SN95 FKS1 $1^{\text {F641S/FKS1 CAS5/cas5::FRT }}$ strain. Correct upstream and downstream integration at the CAS5 locus was verified as described for CaLC2034. The absence of a wild-type allele was confirmed with oLC2034 in combination with oLC2164. The SAP2 promoter was induced to drive expression of FLP recombinase to excise the NAT flipper cassette.

CaLC3378: To C terminally HA tag CAS5 in a mutant carrying the $P K C 1^{M 850 G}$ mutation, the CAS5-HA-HIS cassette was released from pLC790 with ApaI and transformed into SN95 $P K C 1^{M 850 G} / P K C 1$. Proper integration at the CAS5 locus was verified by amplification using primer pairs oLC2163 in combination with oLC2029 and oLC2164 in combination with oLC1645. Expression of HA-tagged Cas5 was verified by western blot.

CaLC3859: To regulate the expression of CAS5, the tetracycline-repressible transactivator, the tet $O$ promoter, and the NAT flipper cassette were PCR amplified from pLC605 ${ }^{61}$ using primers oLC2088 in combination with oLC2089. The PCR amplified product was transformed into CaLC2034. Correct upstream and downstream integration at the CAS5 locus was verified by amplifying across both junctions using primer pairs oLC2034 in combination with oLC534 and oLC300 in combination with oLC2145. The absence of a wildtype CAS5 promoter was verified with oLC2034 in combination with oLC2035. To C terminally HA tag CAS5, the CAS5-HA-HIS1 cassette was released from pLC818 with SacII and transformed into the tetO-CAS5/cas5 $\Delta$. Correct upstream integration at the $\mathrm{C}$ terminus of CAS5 was verified by amplifying across the junction using primer pair oLC2163 in combination with oLC2164. Expression of HA-tagged Cas5 was verified by western blot.

CaLC3113: To C terminally HA tag CAS5 in a mutant heterozygous for CAS5, the HA-HIS1 cassette was PCR amplified from pLC575 ${ }^{62}$ using primers oLC2161 in combination with oLC2162 and transformed into CaLC2034. Transformants prototrophic for histidine were PCR tested with C2163 in combination with oLC2029 for upstream integration and oLC2164 in combination with oLC1645 for downstream integration.

CaLC3151: To C terminally HA tag CAS5 in a mutant heterozygous for CAS5, the CAS5-HA-HIS1 cassette was released from pLC790 with ApaI and transformed into CaLC2034. Transformants prototrophic for histidine were PCR tested with oLC2163 in combination with oLC2029 for upstream integration and oLC2164 in combination with oLC1645 for downstream integration. This strain shares the same genotype as CaLC3113 but was generated as a control for strains made using plasmid pLC790 as the backbone, such pLC791 and pLC800.

CaLC4285: To C terminally HA tag CAS5, the HA-ARG4 cassette was PCR amplified from pLC576 $6^{62}$ using primers oLC2161 in combination with oLC2162 and transformed into CaLC1900. Correct integration at the $\mathrm{C}$ terminus of CAS5 was verified by using primer pair oLC2163 in combination with oLC2164.

CaLC2213: To C terminally HA tag CAS5 in SN95, the HA-HIS1 cassette was PCR amplified from pLC575 $5^{62}$ using primers oLC2161 in combination with oLC2162 and transformed into CaLC239 (SN95). Transformants prototrophic for histidine were PCR tested for correct integration of the cassette with oLC2163 in combination with oLC2029 and oLC2164 in combination with oLC1645.

CaLC3044: To C terminally HA tag the both alleles of CAS5, the HA-ARG4 cassette was PCR amplified from pLC576 ${ }^{62}$ as described for CaLC4285 and transformed into CaLC2213. Transformants prototrophic for both histidine and arginine were PCR tested for correct integration of the cassette as described for CaLC4285.

CaLC3209: To introduce a mutant allele of CAS5 carrying the S769E mutation, the CAS5 ${ }^{\mathrm{S} 769 \mathrm{E}}-$ HA-HIS1 cassette was released from pLC791 with ApaI and transformed into CaLC2034. Transformants prototrophic for histidine were PCR tested for integration of the cassette with oLC3052 in combination with oLC2029 and oLC2164 in combination with oLC1645. The absence of a wildtype CAS5 allele was verified with oLC3052 in combination with oLC2164. The S769E mutation was sequence verified with oLC2029.

CaLC3189: To introduce a mutant allele of CAS5 carrying the S769A mutation, the CAS5 ${ }^{\mathrm{S} 769 \mathrm{~A}}-\mathrm{HA}-H I S 1$ cassette was released from pLC800 with ApaI and transformed into CaLC2034. Transformants prototrophic for histidine were PCR tested for integration of the cassette as described for CaLC3209. The S769A mutation was sequence verified with oLC2029.

CaLC4036: To generate a SWI4 homozygous deletion mutant, the NAT flipper cassette (pLC49) ${ }^{60}$ was PCR amplified using primers oLC3472 in combination with oLC3473 and transformed into CaLC239 (SN95). NAT-resistant transformants were PCR tested with oLC275 in combination with oLC3474 and oLC274 in combination with oLC3427. The SAP2 promoter was induced to drive expression of FLP recombinase to excise the NAT flipper cassette. Once again, the NAT flipper cassette (pLC49) ${ }^{60}$ was PCR amplified using primers oLC3472 in combination with oLC3473 and transformed into SWI4/swi4::FRT. NAT-resistant transformants were PCR tested as described above. The absence of a wild-type allele was verified with oLC3426 in combination with oLC3427, and the presence of deleted allele was verified by oLC3473 in combination with oLC3427. The SAP2 promoter was induced to drive expression of FLP recombinase to excise the NAT flipper cassette.

CaLC4330: To generate a SWI6 homozygous deletion mutant, the NAT flipper cassette (pLC49) ${ }^{60}$ was PCR amplified using primers oLC3641 in combination with oLC3642 and transformed into CaLC239 (SN95). NAT-resistant transformants were PCR tested with oLC275 in combination with oLC3645 and oLC274 in combination with oLC3654. The SAP2 promoter was induced to drive expression of FLP recombinase to excise the NAT flipper cassette. Once again, the NAT flipper cassette (pLC49) ${ }^{60}$ was PCR amplified using primers oLC3641 in combination with oLC3642 and transformed into SWI6/swi6::FRT. NAT-resistant transformants were PCR tested as described above. The absence of a wild-type allele and the presence of the deleted allele was verified with oLC3645 in combination with oLC3431. The SAP2 promoter was induced to drive expression of FLP recombinase to excise the NAT flipper cassette.

CaLC3391: To C terminally TAP tag Swi4, the TAP-ARG4 cassette was PCR amplified from pLC573 ${ }^{62}$ using primers oLC3424 in combination with oLC3425 and transformed into CaLC239. Transformants prototrophic for arginine were PCR tested with oLC3426 in combination with oLC1593 and oLC1594 in combination with oLC3427.

CaLC3393: To C terminally TAP tag Swi6, the TAP-ARG4 cassette was PCR amplified from pLC573 ${ }^{62}$ using primers oLC3428 in combination with oLC3429 and transformed into CaLC239. Transformants prototrophic for arginine were 
PCR tested with oLC3430 in combination with oLC1593 and oLC1594 in combination with oLC3431.

CaLC3395: To C terminally TAP tag Swi4 in a strain with HA-tagged Cas5, the TAP-ARG4 cassette was PCR amplified from pLC573 ${ }^{62}$ as described for CaLC3391 and transformed into CaLC3151. Transformants prototrophic for arginine and histidine were PCR tested as described for CaLC3391.

CaLC3398: To C terminally TAP tag Swi6 in a strain with HA-tagged Cas5, the TAP-ARG4 cassette was PCR amplified from pLC573 ${ }^{62}$ as described for CaLC3393 and transformed into CaLC3151. Transformants prototrophic for arginine and histidine were PCR tested as described for CaLC3393.

CaLC3932: To generate a GLC7 heterozygous deletion mutant, the NAT flipper cassette (pLC49) ${ }^{60}$ was PCR amplified using primers oLC3490 in combination with oLC3491 and transformed into CaLC239 (SN95). NAT-resistant transformants were PCR tested with oLC275 in combination with oLC3558 and oLC274 in combination with oLC3561. The SAP2 promoter was induced to drive expression of FLP recombinase to excise the NAT flipper cassette. To regulate the expression of GLC7, the tetracycline-repressible transactivator, the tet $O$ promoter, and the NAT flipper cassette were PCR amplified from pLC605 $5^{61}$ using primers oLC3793 in combination with oLC3492 and transformed into GLC7/glc7::FRT. NATresistant transformants were PCR tested with oLC3794 in combination with oLC534 and oLC274 in combination with oLC3495. The absence of a wildtype GLC7 promoter was verified with oLC3794 in combination with oLC3495, and the presence of a deleted allele was verified with oLC3794 in combination with oLC3494. The SAP2 promoter was induced to drive expression of FLP recombinase to excise the NAT flipper cassette.

CaLC3952: To C terminally HA tag CAS5 in the GLC7 repressible strain, the HA-HIS1 cassette was PCR amplified from pLC575 ${ }^{62}$ using primers oLC2161 in combination with oLC2162 and transformed into CaLC3932. Transformants prototrophic for histidine were PCR tested with oLC2163 in combination with oLC2164.

CaLC3693/CaLC3694/CaLC3695: To introduce a mutant allele of CAS5 carrying S462E, S464E, S472E, and S476E mutations, the CAS5S S462E/S464E/S472E/ S476E-HA-HIS1 cassette was released from pLC857 with SacII and transformed into CaLC2034. Transformants prototrophic for histidine were PCR tested with oLC3052 in combination with oLC2029 and oLC2164 in combination with oLC1645. The absence of an untagged wild type CAS5 allele was verified with oLC3052 in combination with oLC2164. The S462A/S464A/S472A/S476A mutations were sequence verified with oLC3371.

CaLC3672/CaLC3673: To introduce a mutant allele of CAS5 carrying S462A S464A, S472A, and S476A mutations, the CAS5S $462 \mathrm{~A} / \mathrm{S} 464 \mathrm{~A} / \mathrm{S} 472 \mathrm{~A} / \mathrm{S} 476 \mathrm{~A}-\mathrm{HA}-\mathrm{HIS} 1$ cassette was released from pLC858 with SacII and transformed into CaLC2034. Transformants prototrophic for histidine were PCR tested as described for CaLC3693. The absence of an untagged wildtype CAS5 allele was verified with oLC3052 in combination with oLC2164. The S462A/S464A/S472A/S476A mutations were sequence verified with oLC3371.

CaLC4471: To C terminally TAP tag Swi4 in a mutant heterozygous for SWI4, the TAP-ARG4 cassette was PCR amplified from pLC573 ${ }^{62}$ using primers oLC3424 in combination with oLC3425 and transformed into CaLC3151. Transformants prototrophic for arginine were PCR tested with oLC3426 in combination with oLC3427.

CaLC4499: To C terminally TAP tag Swi6 in a mutant heterozygous for SWI6, the TAP-ARG4 cassette was PCR amplified from pLC573 ${ }^{62}$ using primers oLC3428 in combination with oLC3429 and transformed into SWI6/swi6::FRT.

Transformants prototrophic for arginine were PCR tested with oLC3430 in combination with oLC1593 and oLC3430 in combination with oLC3431.

CaLC4705: To C terminally RFP tag Hhf1 in SN95, the RFP-NAT cassette was PCR amplified from pLC447 using primers oLC4752 in combination with oLC4753 and transformed into CaLC239. Correct integration at the $\mathrm{C}$ terminus of HHF1 was verified by amplifying across both junctions using primer pairs oLC4417 in combination with oLC4754 and oLC274 in combination with oLC4755. To C terminally GFP tag Dad2, the GFP-HIS cassette was PCR amplified from pLC383 ${ }^{63}$ using primers oLC4748 in combination with oLC4749 and transformed into $H H F 1-R F P / H H F 1$. Correct integration at the $\mathrm{C}$ terminus of $D A D 2$ was verified by amplifying across both junctions using primer pairs oLC600 in combination with oLC4750 and oLC1645 in combination with oLC4751.

CaLC4707: To C terminally RFP tag Hhf1 in a cas $5 \Delta /$ cas $5 \Delta$ mutant, the RFPNAT cassette was PCR amplified from pLC447 as described for CaLC4705 and transformed into CaLC2056. Correct integration at the $\mathrm{C}$ terminus of HHF1 was verified as described for CaLC4705. To C terminally GFP tag Dad2, the GFP-HIS cassette was PCR amplified from pLC $383^{63}$ as described for CaLC4705 and transformed into cas $5 \Delta /$ cas $5 \Delta H H F 1-R F P / H H F 1$. Correct integration at the $\mathrm{C}$ terminus of DAD2 was verified by as described for CaLC4705.

Plasmid construction. Cloning procedures were performed following standard protocols. Plasmids used in this study are listed in Supplementary Table 2. The absence of nonsynonymous mutations in plasmids was verified by sequencing. Primers used in this study are listed in Supplementary Table 3. Plasmid construction is described in detail below.

pLC447: This is a construct to tag proteins with RFP at the $\mathrm{C}$ terminus. CaCherry/RFP was PCR amplified from pLC435 ${ }^{64}$ with oLC841 in combination with oLC842 and digested with BsrGI. This was cloned into pLC389 at BsrGI
Directionality of the insert was verified by PCR with pLC849 in combination with oLC842. The construct was sequence verified with oLC849 and oLC842.

pLC790: This is a construct to C terminally HA-tag Cas5 in C. albicans. The CAS5 DNA-binding domain, HA, and HIS marker were amplified from CaLC3113 genomic DNA with primers oLC3092 in combination with oLC3052 and cloned into pLC49 at the ApaI site. The ligation mixture was transformed into TOP10 cells. Cells were plated on LB + Amp + NAT. Correct integration of the cassette in pLC49 was sequence verified with oLC2029. This construct can be liberated by digestion with ApaI and transformed into C. albicans.

pLC791: This plasmid is based on pLC790 but harbors a phosphomimetic mutation in CAS5 (S769E). This mutation was introduced by site-directed mutagenesis with primers oLC2986 and oLC2987. The clone was sequence verified with oLC2029. This construct can be liberated by digestion with ApaI and transformed into C. albicans.

pLC800: This plasmid is based on pLC790 but harbors a phosphomimetic mutation in CAS5 (S772E). This mutation was introduced by site-directed mutagenesis with primers oLC2990 and oLC2991. The plasmid was sequence verified with oLC2029. This construct can be liberated by digestion with ApaI and transformed into C. albicans.

pLC818: This is a construct to C terminally HA-tag Cas5 in C. albicans. The CAS5 ORF, HA, and HIS1 were amplified from CaLC3113 genomic DNA with primers oLC3365 in combination with oLC3366 and cloned into pLC49 at the SacII site. The ligation mixture was transformed into TOP10 cells. Cells were plated on LB + Amp + NAT. Correct integration of the cassette in pLC49 was sequence verified with oLC2029, oLC244, and oLC3371. This construct can be liberated by digestion with SacII and transformed into C. albicans.

pLC828: This plasmid is based on pLC818 but harbors a phosphomimetic mutation in CAS5 (S472E/S476E). This mutation was introduced by site-directed mutagenesis with primers oLC3410 and oLC3411. The plasmid was sequence verified with oLC3371. This construct can be liberated by digestion with SacII and transformed into C. albicans.

pLC857: This plasmid is based on pLC828 but harbors additional phosphomimetic mutations in CAS5 (S462E/S464E). This mutation was introduced by site-directed mutagenesis with primers oLC3416 and oLC3417. The plasmid was sequence verified with oLC3371. This construct can be liberated by digestion with SacII and transformed into C. albicans.

pLC833: This plasmid is based on pLC818 but harbors a phosphodeficient mutation in CAS5 (S472A/S476A). This mutation was introduced by site-directed mutagenesis with primers oLC3412 and oLC3413. The clone was sequence verified with oLC3371. This construct can be liberated by digestion with SacII and transformed into C. albicans.

pLC858: This plasmid is based on pLC833 but harbors additional phosphodeficient mutations in CAS5 (S462A/S464A). This mutation was introduced by site-directed mutagenesis with primers oLC3418 and oLC3419. The plasmid was sequence verified with oLC3371. This construct can be liberated by digestion with SacII and transformed into C. albicans.

Culturing conditions. All cultures were grown shaking at $200 \mathrm{rpm}$. To assess the effects of caspofungin and calcofluor white treatment on the expression of Cas5 and Cas5-dependent of transcripts, overnight cultures were diluted to $\mathrm{OD}_{600}$ of $0.15 \mathrm{in} \mathrm{YPD} \mathrm{for} 3 \mathrm{~h}$, and either untreated or treated with $125 \mathrm{ng} / \mathrm{ml}$ of caspofungin or $100 \mu \mathrm{g} / \mathrm{ml}$ of calcofluor white for 1 or $2 \mathrm{~h}$, as specified. To deplete GLC7, strains were grown overnight at $30^{\circ} \mathrm{C}$ in YPD. Stationary phase cultures were split, adjusted to an $\mathrm{OD}_{600}$ of 0.15 , at which point one culture was treated with $0.02 \mu \mathrm{g} /$ $\mathrm{ml}$ of doxycycline, whereas the other was left untreated. Cells were grown for $16 \mathrm{~h}$ and were split again, adjusted to an $\mathrm{OD}_{600}$ of 0.15 and grown for $3 \mathrm{~h}$. One culture was subsequently treated with $125 \mathrm{ng} / \mathrm{ml}$ of caspofungin for 1 or $2 \mathrm{~h}$ as specified, whereas the other was left untreated.

ChIP-Seq data generation and analysis. Chromatin preparation and immunoprecipitation against PolII using $2 \mu$ of 8 WG16 antibody were performed as described previously ${ }^{65}$. Multiplex sequencing libraries were constructed according to Wong et al. ${ }^{66}$ ChIP-Seq libraries were assessed by Bioanalyzer DNA High Sensitivity Assay and quantified using real-time PCR before sequencing on the Illumina HiSeq2500 platform. Raw reads were mapped to the C. albicans reference genome. Mapped reads on gene bodies were counted, normalized to total number of mapped reads and expressed as "Normalized PolII ChIP-Seq signal". Differentially bound genes are defined by greater than 1.5-fold difference in PolII ChIP-Seq signals between any two conditions analyzed. Non-expressed genes or lowly expressed genes (e.g., genes with PolII ChIP-Seq signals lower than 10) under both conditions analyzed were removed from downstream analysis. Pathway analysis was carried out using the GO program on the Candida Genome Database (http:// www.candidagenome.org/cgi-bin/GO/goTermFinder) and the KEGG mapper (http://www.genome.jp/kegg/tool/map_pathway2.html).

Minimum inhibitory concentration assay. Antifungal susceptibility was measured in flat bottom, 96-well microtitre plates (Sarstedt \#83.3924) using a broth microdilution protocol described in ref. ${ }^{67}$. In brief, minimum inhibitory concentration (MIC) assays were set up in twofold serial dilutions of caspofungin or calcofluor white in a final volume of $200 \mu \mathrm{l}$ per well. Caspofungin gradients were diluted 
either from $125 \mathrm{ng} / \mathrm{ml}$ down to $0.12 \mathrm{ng} / \mathrm{ml}$ or 2000 to $3.9 \mathrm{ng} / \mathrm{ml}$. Calcofluor white gradients were diluted from $250 \mu \mathrm{g} / \mathrm{ml}$ down to $0.24 \mu \mathrm{g} / \mathrm{ml}$. Where applicable, doxycycline was added to a final concentration of $0.1 \mu \mathrm{g} / \mathrm{ml}$. Cell densities of overnight cultures were determined and dilutions were prepared such that $\sim 10^{3}$ cells were inoculated into each well. Plates were incubated in the dark at $30^{\circ} \mathrm{C}$ for $48 \mathrm{~h}$, at which point the absorbance was determined at $600 \mathrm{~nm}$ using a spectrophotometer (Molecular Devices) and corrected for background from the corresponding medium. Each strain was tested in duplicate in three biological replicates. MIC data was quantitatively displayed with color using the program Java TreeView 1.1.3 (http://jtreeview.sourceforge.net).

Flow cytometry analysis. Yeast strains were cultured overnight in $4 \mathrm{ml}$ YPD at $30{ }^{\circ} \mathrm{C}$. Cells were subcultured for $4-5 \mathrm{~h}$ and isolated during exponential growth Overall, $400 \mu \mathrm{l}$ of cells were pelleted and fixed overnight in $70 \%$ ethanol at $4{ }^{\circ} \mathrm{C}$ The fixed cells were washed twice with $1 \mathrm{ml}$ of sodium citrate $(50 \mathrm{mM})$ and resuspended in $500 \mu \mathrm{l}$ sodium citrate and $12 \mu \mathrm{l}$ of RNaseA $(25 \mathrm{mg} / \mathrm{ml})$. The cells were incubated at $37^{\circ} \mathrm{C}$ for $4 \mathrm{~h}$. Then $17 \mu \mathrm{l}$ of propidium iodide was added to the RNase-treated cells and incubated overnight at $37^{\circ} \mathrm{C}$. The cells were vortexed and sonicated for $5 \mathrm{~s}$ at level 15 of the Sonic Desmembrator (Fisher Scientific), and filtered immediately before analysis using a BD Falcon tube with a $35 \mu \mathrm{m}$ nylon mesh cap (Cat. No. 352235). Ploidy was determined using a Yetti flow cytometer, and 50,000-100,000 cells were analyzed per strain.

Co-immunoprecipitation. Cultures were grown to mid-exponential phase and harvested by centrifugation at $3000 \mathrm{rpm}$ for $5 \mathrm{~min}$. The cells were washed with sterile $\mathrm{H}_{2} \mathrm{O}$ and resuspended in $1 \mathrm{~mL}$ of lysis buffer $(20 \mathrm{mM}$ Tris $\mathrm{pH} 7.5,100 \mathrm{mM}$ $\mathrm{KCl}, 5 \mathrm{mM} \mathrm{MgCl}$, and $20 \%$ glycerol), with one protease inhibitor cocktail per $50 \mathrm{ml}$ (complete, EDTA-free tablet, Roche Diagnostics, Indianapolis, IN, USA) and $1 \mathrm{mM}$ PMSF (EMD Chemicals, Gibbstown, NJ, USA). Cells were lysed by bead beating twice for $4 \mathrm{~min}$ with $7 \mathrm{~min}$ on ice between cycles. Lysates were recovered by piercing a hole in the bottom of each tube, placing each tube in a $14 \mathrm{ml}$ conical tube, and centrifuging at $1300 \times g$ for three 5 -min cycles, recovering the supernatant after each stage. The combined lysate was cleared by centrifugation at $21,000 \times g$ for $10 \mathrm{~min}$ at $4{ }^{\circ} \mathrm{C}$. The protein concentrations were determined using the Bradford assay ${ }^{68}$. Anti-HA immunoprecipitations were done using Pierce HA-Tag IP/Co-IP kit (Thermo-Fisher Scientific, PI23610), as per manufacturer's instructions.

Western blot analysis. Protein was extracted by pelleting cells at $\mathrm{OD}_{600}$ of 0.8 , with the pellet being resuspended in $2 \times$ sample buffer (one-third volume of $6 \times$ sample buffer containing $0.35 \mathrm{M}$ Tris- $\mathrm{HCl}, 10 \%(\mathrm{w} / \mathrm{w})$ SDS, $36 \%$ glycerol, $5 \% \beta$ mercaptoethanol, and $0.012 \%$ bromophenol blue). The samples were boiled for 5 $\min$ at $95^{\circ} \mathrm{C}$. The cell debris was pelleted and the supernatant was separated on a $6 \%$ SDS-PAGE gel to observe changes in Cas5 mobility. Separated proteins were electrotransferred to PVDF membrane (Bio-Rad Laboratories) and blocked with $5 \%$ skim milk in phosphate-buffered saline (PBS) with $0.2 \%$ Tween-20 at room temperature for $1 \mathrm{~h}$. Blots were hybridized with antibody against the HA epitope (1:5000 dilution; Roche Diagnostics), p44/42 (1:2000, Cell Signaling), PSTAIRE (1:5000, Santa Cruz Biotechnology), TAP (1:5000; Open Biosystems), Hsp90 (1:10,000; generously provided by Brian Larsen, Des Moines University) or $\alpha-\beta$ actin (1:5000; Santa Cruz, sc-47778) overnight at $4{ }^{\circ} \mathrm{C}$. Blots were washed with PBS with $0.1 \%$ Tween- 20 and subsequently hybridized with FITC-conjugated secondary antibody diluted 1:5000 in the block solution for $45 \mathrm{~min}$ at room temperature. Signals were detected using an ECL western blotting kit as per the manufacturer's instructions (Pierce).

Two-dimensional western blot analysis. C. albicans Cas5-HA cells were harvested from log phase cultures in YPD (+/-caspofungin) with lysis buffer $(150 \mathrm{mM}$ $\mathrm{NaCl}, 50 \mathrm{mM}$ Tris-HCl pH 7, $15 \mathrm{mM}$ EDTA, 1\% Triton 100-X, 10\% glycerol) plus phosphatase inhibitors (Sigma, P5726 and P0044) and protease inhibitors (Roche complete Mini Protease Inhibitor Cocktail Tablets 04693124001) using glass beads and bead beating $10 \times 20 \mathrm{~s}$ with $1 \mathrm{~min}$ ice in between. Cell extracts were precipitated with methanol-chloroform and resuspended in rehydration buffer (BioRad Cat\#163-2105). Approximately $30 \mu \mathrm{g}$ protein was loaded onto $7 \mathrm{~cm}$ IPG strips (pH 3-10, Bio-Rad Cat\#163-2000) and resolved using Bio-Rad Protean i12 IEF System. The IPG strips were then resolved using $10 \%$ SDS-PAGE gels and processed for western blotting following the procedures described above for one-dimensional western blot analysis.

Quantitative RT-PCR. To prepare samples for RNA extraction, $10 \mathrm{ml}$ of subculture was harvested by centrifugation at $1300 \times \mathrm{g}$ for $5 \mathrm{~min}$. The pellet was flashfrozen and stored at $-80^{\circ} \mathrm{C}$ overnight. RNA was isolated using the QIAGEN RNeasy kit and cDNA was generated using the AffinityScript cDNA synthesis kit (Stratagene). qRT-PCR was carried out using the Fast SYBR Green Master Mix (Thermo-Fisher Scientific) in 384-well plates with the following cycle conditions: $95^{\circ} \mathrm{C}$ for $10 \mathrm{~min}$, repeat $95^{\circ} \mathrm{C}$ for $10 \mathrm{~s}, 60^{\circ} \mathrm{C}$ for $30 \mathrm{~s}$ for 40 cycles. The melt curve was completed with the following cycle conditions: $95^{\circ} \mathrm{C}$ for $10 \mathrm{~s}$ and $65^{\circ} \mathrm{C}$ for $5 \mathrm{~s}$ with an increase of $0.5^{\circ} \mathrm{C}$ per cycle up to $95^{\circ} \mathrm{C}$. All reactions were done in triplicate. Data were analyzed in the Bio-Rad CFX manager 3.1. Data was plotted using GraphPad Prism.
Affinity purification and liquid chromatography-tandem mass spectrometry analysis. For affinity purification (AP) and liquid chromatography-tandem mass spectrometry (LC-MS/MS) analysis, clarified whole-cell lysates $(10 \mathrm{mg}$ protein per sample) were extracted with immobilized anti-HA beads (Pierce HA-Tag IP/Co-IP kit, Thermo-Fisher Scientific, PI23610). After a 3-h incubation, the beads were washed three times with lysis buffer and twice with HPLC water. The proteins bound on beads were eluted with $150 \mu \mathrm{l}$ of $0.15 \%$ trifluoroacetic acid (TFA), neutralized with $100 \mathrm{mM} \mathrm{NH}_{4} \mathrm{HCO}_{3}$, and digested with trypsin. The tryptic peptides were purified by $200 \mu \mathrm{l}$ C18 stage tips (Thermo Scientific, Rockford, IL, USA) and analyzed by Q-Exactive LC-MS/MS ${ }^{69}$. The tryptic peptides from anti-HA IP complexes were separated on a 50-cm Easy-Spray column with a 75- $\mu \mathrm{m}$ inner diameter packed with $2 \mu \mathrm{m} \mathrm{C18}$ resin (Thermo Scientific, Odense, Denmark). The peptides were eluted over $120 \mathrm{~min}(250 \mathrm{nl} / \mathrm{min})$ using a 0 to $40 \%$ acetonitrile gradient in $0.1 \%$ formic acid with an EASY nLC 1000 chromatography system operating at $50{ }^{\circ} \mathrm{C}$ (Thermo-Fisher Scientific). The LC was coupled to a Q-Exactive mass spectrometer ${ }^{70}$ by using a nano-ESI source (Thermo-Fisher Scientific). Mass spectra were acquired in a data-dependent mode with an automatic switch between a full scan and up to 10 data-dependent MS/MS scans. Target value for the full scan MS spectra was $1 \mathrm{e} 6$ with a maximum injection time of $120 \mathrm{~ms}$ and a resolution of 70,000 at $\mathrm{m} / \mathrm{z} 400$. The ion target value for MS/MS was set to $1,000,000$ with a maximum injection time of $120 \mathrm{~ms}$ and a resolution of 17,500 at $\mathrm{m} / \mathrm{z} 400$. The first mass for the MS/MS was set to $140 \mathrm{~m} / z$ and the normalized collision energy was set to 27. Unassigned, as well as charge states 1 and $>5$ were ignored for MS/MS selection. Repeat sequencing of peptides was kept to a minimum by dynamic exclusion of sequenced peptides for $12 \mathrm{~s}^{71}$.

Acquired raw files were analyzed by using MaxQuant software ${ }^{72}$ (version 1.3.0.5) for quantification, and X! Tandem (The GPM, thegpm.org; version CYCLONE; 2010.12.01.1) and Scaffold (version Scaffold_3.4.3, Proteome Software, Portland, OR, USA) for further validation. The default search parameters were used as described by Deeb et al. ${ }^{71}$. The search included cysteine carbamidomethylation as a fixed modification, as well as $\mathrm{N}$-terminal acetylation, methionine oxidation, and phospho-serine, -threonine, and -tyrosine as variable modifications. Localization probability for phosphorylation sites was required to exceed $75 \%$. The second peptide identification option in Andromeda was enabled. For statistical evaluation of the data obtained, the posterior error probability and false discovery rate were used. The false discovery rate was determined by searching a reverse database. A false discovery rate of 0.01 for proteins and peptides was permitted. Two miscleavages were allowed, and a minimum of seven amino acids per identified peptide were required. Peptide identification was based on a search with an initial mass deviation of the precursor ion of up to $6 \mathrm{ppm}$, and the allowed fragment mass deviation was set to $20 \mathrm{ppm}$. To match identifications across different replicates and adjacent fractions, the "match between runs" option in MaxQuant was enabled within a time window of $2 \mathrm{~min}$.

Microscopic analysis. Cultures were grown to mid-exponential phase and $1 \mathrm{ml}$ of the cells was centrifuged at 14,000 rpm for $1 \mathrm{~min}$. The supernatant was removed and the cells were washed with PBS. To stain for chitin, calcofluor white was added to a final concentration of $1 \mu \mathrm{g} / \mathrm{ml}$ in a final volume of $100 \mu \mathrm{l}$. The cells were incubated in the dark for $15 \mathrm{~min}$ and were gently vortexted every 3-4 min. Cells were washed with PBS and $2 \mu \mathrm{l}$ of the cells were deposited on a cover slide. To stain for nuclei, the cells were heat fixed on the slide for $1 \mathrm{~min}$ at $75^{\circ} \mathrm{C}$ and $0.5 \mu \mathrm{l}$ of 1 $\mathrm{mg} / \mathrm{ml}$ DAPI was added. All imaging was performed on a Zeiss Imager M1 uprigh microscope and AxioCam Mrm with AxioVision 4.7 software. For fluorescence microscopy, an X-cite series 120 light source with ET green fluorescent protein (GFP), 4',6-diamidino-2-phenylindole (DAPI) hybrid, and ET HQ tetramethylrhodamine isothiocyanate (TRITC)/DsRED filter sets from Chroma Technology (Bellows Falls, VT, USA) was used. Calcofluor white and DAPI were viewed under the DAPI hybrid filter and HA-tagged Cas5 was viewed under the Texas Red filter. To facilitate nuclei counting, $12 \mathrm{Z}$ stacks were taken for each image, where each slice is $0.3 \mu \mathrm{m}$.

Indirect immunofluorescence microscopy. To obtain cells for immunofluorescence, $5-10 \mathrm{ml}$ of subculture was grown to mid-exponential phase and fixed with $5 \%$ formaldehyde for $3-4 \mathrm{~h}$ at $30^{\circ} \mathrm{C}$. Cells were harvested at $1300 \times g$ for $5 \mathrm{~min}$ and washed once with $5 \mathrm{ml} \mathrm{S-Buffer} \mathrm{(50} \mathrm{mM} \mathrm{HEPES} \mathrm{pH} \mathrm{7.5,} \mathrm{1.2} \mathrm{M} \mathrm{Sorbitol)} \mathrm{before}$ being resuspended in $1 \mathrm{ml} \mathrm{S-Buffer}$. To induce spheroplast formation, $10 \mu \mathrm{l}$ of $1 \mathrm{M}$ DTT, $30 \mu \mathrm{l}$ of glusulase, and $40 \mu \mathrm{l}$ of $2.5 \mathrm{mg} / \mathrm{ml}$ zymolase were added to the cells and the mix was incubated for $30 \mathrm{~min}$ at $37^{\circ} \mathrm{C}$. The extent of spheroplasting was monitored under the microscope. The poly lysine (Lys) coated slides were prepared by adding $15 \mu \mathrm{l}$ of $0.1 \%$ poly Lys per slide well and set aside for $15 \mathrm{~min}$. Wells were washed three times with PBS. The fixed cells were centrifuged for $1 \mathrm{~min}$ at 5000 $\mathrm{rpm}$ and gently resuspended in $1 \mathrm{ml} \mathrm{S-buffer}$. Following the addition of $0.1 \%$ Triton X-100, the mix was incubated for $5 \mathrm{~min}$ on rocker. The cells were centrifuged for $1 \mathrm{~min}$ at $5000 \mathrm{rpm}$ and resuspended in $1 \mathrm{ml} \mathrm{S}$-Buffer. To adhere the cells, $20 \mu \mathrm{l}$ of cell suspension was added on to poly Lys-coated well and incubated it for $15 \mathrm{~min}$. The cells were washed three times with PBS-T and the wells were blocked with $20 \mu \mathrm{l}$ PBS/BSA for 5-10 min. Anti-HA antibody diluted 1:300 in $20 \mu \mathrm{l}$ was added and incubated for $3-4 \mathrm{~h}$ or overnight in a humid chamber. The cells were washed four times in PBS-T. Anti-mouse IgG-Cy3 diluted 1:500 in PBS-T was added to the wells and incubated for $1-2 \mathrm{~h}$. The cells were washed four times with 
PBS-T and once with only PBS. Overall, $20 \mu \mathrm{l} \mathrm{of} 1 \mathrm{mg} / \mathrm{ml}$ DAPI diluted 1:1000 in PBS was added to the wells and incubated for $5 \mathrm{~min}$. The cells were washed four times with PBS. The cells were left to dry in the dark for $30 \mathrm{~min}$ at room temperature. Mounting medium was added and cells were viewed under the microscope.

Data availability. The ChIP-seq data have been deposited in the NCBI Sequence Read Archive with accession code SRP106998. The authors declare that all other data supporting the findings of the study are available in this article and its Supplementary Information files, or from the corresponding author upon request.

Received: 3 March 2017 Accepted: 6 July 2017

Published online: 11 September 2017

\section{References}

1. Falagas, M. E., Roussos, N. \& Vardakas, K. Z. Relative frequency of albicans and the various non-albicans Candida spp among candidemia isolates from inpatients in various parts of the world: a systematic review. Int. J. Infect. Dis. 14, e954-966 (2010).

2. Pfaller, M. A. \& Diekema, D. J. Epidemiology of invasive candidiasis: a persistent public health problem. Clin. Microbiol. Rev. 20, 133-163 (2007).

3. Sanchez-Vargas, L. O. et al. Oral Candida isolates colonizing or infecting human immunodeficiency virus-infected and healthy persons in Mexico. J. Clin. Microbiol. 43, 4159-4162 (2005).

4. Southern, P., Horbul, J., Maher, D. \& Davis, D. A. C. albicans colonization of human mucosal surfaces. PLoS ONE 3, e2067 (2008).

5. Kim, J. \& Sudbery, P. Candida albicans, a major human fungal pathogen. J. Microbiol. 49, 171-177 (2011).

6. Mayer, F. L., Wilson, D. \& Hube, B. Candida albicans pathogenicity mechanisms. Virulence 4, 119-128 (2013).

7. Calderone R. A. \& Clancy C. J. Candida and Candidiasis. 225-242 (ASM Press, 2012).

8. Singh, S. D. et al. Hsp90 governs echinocandin resistance in the pathogenic yeast Candida albicans via calcineurin. PLoS Pathog. 5, e1000532 (2009).

9. LaFayette, S. L. et al. PKC signaling regulates drug resistance of the fungal pathogen Candida albicans via circuitry comprised of Mkcl, calcineurin, and Hsp90. PLoS Pathog. 6, e1001069 (2010).

10. Chamilos, G. et al. Candida albicans Cas5, a regulator of cell wall integrity, is required for virulence in murine and toll mutant fly models. J. Infect. Dis. 200, 152-157 (2009).

11. Cannon, R. D. et al. Candida albicans drug resistance another way to cope with stress. Microbiology 153, 3211-3217 (2007).

12. The U.S. Centers for Disease Control and Prevention. Antibiotic Resistance Threats in the United States, 2013. 112 (CDC, 2013).

13. Mishra, N. N. et al. Pathogenicity and drug resistance in Candida albicans and other yeast species. A review. Acta Microbiol. Immunol. Hung. 54, 201-235 (2007).

14. Ruggero, M. A. \& Topal, J. E. Development of echinocandin-resistant Candida albicans candidemia following brief prophylactic exposure to micafungin therapy. Transpl. Infect. Dis. 16, 469-472 (2014).

15. Marco, F., Pfaller, M. A., Messer, S. A. \& Jones, R. N. Activity of MK-0991 (L-743,872), a new echinocandin, compared with those of LY303366 and four other antifungal agents tested against blood stream isolates of Candida spp. Diagn. Microbiol. Infect. Dis. 32, 33-37 (1998).

16. Walker, L. A., Gow, N. A. \& Munro, C. A. Fungal echinocandin resistance. Fungal Genet. Biol. 47, 117-126 (2010).

17. Park, S. et al. Specific substitutions in the echinocandin target Fks1p account for reduced susceptibility of rare laboratory and clinical Candida sp. isolates. Antimicrob. Agents Chemother. 49, 3264-3273 (2005).

18. Perlin, D. S. Resistance to echinocandin-class antifungal drugs. Drug Resist. Update 10, 121-130 (2007).

19. Stathopoulos-Gerontides, A., Guo, J. J. \& Cyert, M. S. Yeast calcineurin regulates nuclear localization of the Crzlp transcription factor through dephosphorylation. Genes Dev. 13, 798-803 (1999).

20. Santos, M. \& de Larrinoa, I. F. Functional characterization of the Candida albicans CRZ1 gene encoding a calcineurin-regulated transcription factor. Curr. Genet. 48, 88-100 (2005).

21. Jung, U. S., Sobering, A. K., Romeo, M. J. \& Levin, D. E. Regulation of the yeast Rlm1 transcription factor by the Mpk1 cell wall integrity MAP kinase. Mol. Microbiol. 46, 781-789 (2002).

22. Bruno, V. M. et al. Control of the C. albicans cell wall damage response by transcriptional regulator Cas5. PLoS Pathog. 2, e21 (2006).

23. Nadal, E. D. \& Posas, F. in Stress Response in Microbiology (ed. Requena, J. M.) Ch. 11, 257-285 (Caister Academic Press, 2012)
24. Ene, I. V. et al. Cell wall remodeling enzymes modulate fungal cell wall elasticity and osmotic stress resistance. mBio. 6, e00986 (2015).

25. Heilmann, C. J. et al. Surface stress induces a conserved cell wall stress response in the pathogenic fungus Candida albicans. Eukaryot. Cell 12, 254-264 (2013).

26. Zapater, M., Clotet, J., Escote, X. \& Posas, F. Control of cell cycle progression by the stress-activated Hog1 MAPK. Cell Cycle 4, 6-7 (2005).

27. Leach, M. D. et al. Hsp90 orchestrates transcriptional regulation by Hsf1 and cell wall remodelling by MAPK signalling during thermal adaptation in a pathogenic yeast. PLoS Pathog. 8, e1003069 (2012).

28. Eisman, B. et al. The Cek1 and Hog1 mitogen-activated protein kinases play complementary roles in cell wall biogenesis and chlamydospore formation in the fungal pathogen Candida albicans. Eukaryot. Cell 5, 347-358 (2006).

29. Levin, D. E. Regulation of cell wall biogenesis in Saccharomyces cerevisiae: the cell wall integrity signaling pathway. Genetics 189, 1145-1175 (2011).

30. Mokry, M. et al. Integrated genome-wide analysis of transcription factor occupancy, RNA polymerase II binding and steady-state RNA levels identify differentially regulated functional gene classes. Nucleic Acids Res. 40, 148-158 (2012).

31. Han, Z. et al. A signal processing approach for enriched region detection in RNA polymerase II ChIP-seq data. BMC Bioinformatics 13, S2 (2012).

32. Navarro-Garcia, F., Sanchez, M., Pla, J. \& Nombela, C. Functional characterization of the $M K C 1$ gene of Candida albicans, which encodes a mitogen-activated protein kinase homolog related to cell integrity. Mol. Cell. Biol. 15, 2197-2206 (1995)

33. Cote, P., Hogues, H. \& Whiteway, M. Transcriptional analysis of the Candida albicans cell cycle. Mol. Biol. Cell 20, 3363-3373 (2009).

34. Tye, B. K. MCM proteins in DNA replication. Annu. Rev. Biochem. 68, 649-686 (1999).

35. Xie, J. L. et al. Signaling through Lrg1, Rho1 and Pkc1 governs Candida albicans morphogenesis in response to diverse cues. PLoS Genet. 12, e1006405 (2016).

36. Gould, C. M. et al. Active site inhibitors protect protein kinase C from dephosphorylation and stabilize its mature form. J. Biol. Chem. 286, 28922-28930 (2011).

37. Whitmarsh, A. J. \& Davis, R. J. Regulation of transcription factor function by phosphorylation. Cell. Mol. Life Sci. 57, 1172-1183 (2000).

38. Inglis, D. O. et al. The Candida genome database incorporates multiple Candida species: multispecies search and analysis tools with curated gene and protein information for Candida albicans and Candida glabrata. Nucleic Acids Res. 40, D667-674 (2012).

39. Blom, N., Sicheritz-Ponten, T., Gupta, R., Gammeltoft, S. \& Brunak, S. Prediction of post-translational glycosylation and phosphorylation of proteins from the amino acid sequence. Proteomics 4, 1633-1649 (2004).

40. Gish, W. \& States, D. J. Identification of protein coding regions by database similarity search. Nat. Genet. 3, 266-272 (1993).

41. Lenssen, E. et al. The Ccr4-Not complex independently controls both Msn2-dependent transcriptional activation-via a newly identified Glc7/Bud14 type I protein phosphatase module--and TFIID promoter distribution. Mol. Cell Biol. 25, 488-498 (2005).

42. Schuller, H. J. Transcriptional control of nonfermentative metabolism in the yeast Saccharomyces cerevisiae. Curr. Genet. 43, 139-160 (2003).

43. Andrews, P. D. \& Stark, M. J. Type 1 protein phosphatase is required for maintenance of cell wall integrity, morphogenesis and cell cycle progression in Saccharomyces cerevisiae. J. Cell. Sci. 113, 507-520 (2000).

44. Choi, H. et al. SAINT: probabilistic scoring of affinity purification-mass spectrometry data. Nat. Methods 8, 70-73 (2011).

45. Andrews, B. J. \& Moore, L. A. Interaction of the yeast Swi4 and Swi6 cell cycle regulatory proteins in vitro. Proc. Natl Acad. Sci. USA 89, 11852-11856 (1992).

46. Hussein, B. et al. G1/S transcription factor orthologues Swi4p and Swi6p are important but not essential for cell proliferation and influence hyphal development in the fungal pathogen Candida albicans. Eukaryot. Cell 10, 384-397 (2011)

47. Koch, C., Schleiffer, A., Ammerer, G. \& Nasmyth, K. Switching transcription on and off during the yeast cell cycle: $\mathrm{Cln} / \mathrm{Cdc} 28$ kinases activate bound transcription factor SBF (Swi4/Swi6) at start, whereas Clb/Cdc28 kinases displace it from the promoter in G2. Genes Dev. 10, 129-141 (1996).

48. Harrison, B. D. et al. A tetraploid intermediate precedes aneuploid formation in yeasts exposed to fluconazole. PLoS Biol. 12, e1001815 (2014).

49. Negishi, T. \& Ohya, Y. The cell wall integrity checkpoint: coordination between cell wall synthesis and the cell cycle. Yeast 27, 513-519 (2010).

50. Sadeh, A., Movshovich, N., Volokh, M., Gheber, L. \& Aharoni, A. Fine-tuning of the Msn2/4-mediated yeast stress responses as revealed by systematic deletion of Msn2/4 partners. Mol. Biol. Cell 22, 3127-3138 (2011). 
51. Nicholls, S. et al. Msn2- and Msn4-like transcription factors play no obvious roles in the stress responses of the fungal pathogen Candida albicans. Eukaryot. Cell 3, 1111-1123 (2004).

52. Vasicek, E. M. et al. Disruption of the transcriptional regulator Cas5 results in enhanced killing of Candida albicans by fluconazole. Antimicrob. Agents Chemother. 58, 6807-6818 (2014).

53. Kono, K., Al-Zain, A., Schroeder, L., Nakanishi, M. \& Ikui, A. E. Plasma membrane/cell wall perturbation activates a novel cell cycle checkpoint during G1 in Saccharomyces cerevisiae. Proc. Natl Acad. Sci. USA 113, 6910-6915 (2016).

54. Iyer, V. R. et al. Genomic binding sites of the yeast cell-cycle transcription factors SBF and MBF. Nature 409, 533-538 (2001).

55. Cowen, L. E. The fungal Achilles' heel: targeting Hsp90 to cripple fungal pathogens. Curr. Opin. Microbiol. 16, 377-384 (2013)

56. Cowen, L. E. et al. Harnessing Hsp90 function as a powerful, broadly effective therapeutic strategy for fungal infectious disease. Proc. Natl Acad. Sci. USA 106, 2818-2823 (2009).

57. Pukkila-Worley, R., Peleg, A. Y., Tampakakis, E. \& Mylonakis, E. Candida albicans hyphal formation and virulence assessed using a Caenorhabditis elegans infection model. Eukaryot. Cell 8, 1750-1758 (2009).

58. Nishikawa, J. L. et al. Inhibiting fungal multidrug resistance by disrupting an activator-mediator interaction. Nature 530, 485-489 (2016).

59. Bahn, Y. S. Exploiting fungal virulence-regulating transcription factors as novel antifungal drug targets. PLoS Pathog. 11, e1004936 (2015).

60. Morschhauser, J., Michel, S. \& Staib, P. Sequential gene disruption in Candida albicans by FLP-mediated site-specific recombination. Mol. Microbiol. 32, 547-556 (1999).

61. Leach, M. D. \& Cowen, L. E. Membrane fluidity and temperature sensing are coupled via circuitry comprised of Ole1, Rsp5, and Hsf1 in Candida albicans. Eukaryot. Cell 13, 1077-1084 (2014).

62. Lavoie, H., Sellam, A., Askew, C., Nantel, A. \& Whiteway, M. A toolbox for epitope-tagging and genome-wide location analysis in Candida albicans. BMC Genomics 9, 578 (2008)

63. Gerami-Nejad, M., Berman, J. \& Gale, C. A. Cassettes for PCR-mediated construction of green, yellow, and cyan fluorescent protein fusions in Candida albicans. Yeast 18, 859-864 (2001).

64. Keppler-Ross, S., Noffz, C. \& Dean, N. A new purple fluorescent color marker for genetic studies in Saccharomyces cerevisiae and Candida albicans. Genetics 179, 705-710 (2008).

65. Leach, M. D. et al. Hsfl and Hsp90 orchestrate temperature-dependent global transcriptional remodelling and chromatin architecture in Candida albicans. Nat. Commun. 7, 11704 (2016).

66. Wong, K. H., Jin, Y. \& Moqtaderi, Z. Multiplex Illumina sequencing using DNA barcoding. Curr. Protoc. Mol. Biol. 101, 7.11.1-7.11.11 (2013).

67. Xie, J. L., Singh-Babak, S. D. \& Cowen, L. E. Minimum inhibitory concentration (MIC) assay for antifungal drugs. Bio-Protocol 2, e252 (2012).

68. Bradford, M. M. A rapid and sensitive method for the quantitation of microgram quantities of protein utilizing the principle of protein-dye binding. Anal. Biochem. 72, 248-254 (1976).

69. Tong, J., Taylor, P., Peterman, S. M., Prakash, A. \& Moran, M. F. Epidermal growth factor receptor phosphorylation sites Ser991 and Tyr998 are implicated in the regulation of receptor endocytosis and phosphorylations at Ser1039 and Thr1041. Mol. Cell. Proteom. 8, 2131-2144 (2009).

70. Michalski, A. et al. Mass spectrometry-based proteomics using Q Exactive, a high-performance benchtop quadrupole Orbitrap mass spectrometer. Mol. Cell. Proteom. 10, M111 011015 (2011).

71. Deeb, S. J., D’Souza, R. C., Cox, J., Schmidt-Supprian, M. \& Mann, M. Super-SILAC allows classification of diffuse large B-cell lymphoma subtypes by their protein expression profiles. Mol. Cell. Proteom. 11, 77-89 (2012).
72. Cox, J. \& Mann, M. MaxQuant enables high peptide identification rates, individualized p.p.b.-range mass accuracies and proteome-wide protein quantification. Nat. Biotechnol. 26, 1367-1372 (2008).

\section{Acknowledgements}

We thank Cowen lab members for helpful discussions. We also thank David Rogers (University of Tennessee) for sharing microarray analysis of the CAS5 homozygous mutant, and Li Ang (University of Macau) for assistance in optimizing the ChIP-Seq experiments. J.L.X. is supported by a Canadian Institutes of Health Research Doctoral award and M.D.L. is supported by a Sir Henry Wellcome Postdoctoral Fellowship (Wellcome Trust 096072). B.T.G. holds an Ontario Graduate Scholarship. C.B. and B.J.A are supported by the Canadian Institutes of Health Research Foundation Grants (FDN-143264 and -143265). D.J.K. is supported by a National Institute of Allergy and Infectious Diseases grant (1R01AI098450) and J.D.L.C.D. is supported by the University of Rochester School of Dentistry and Medicine PREP program (R25 GM064133). A.S. is supported by the Creighton University and the Nebraska Department of Health and Human Services (LB506-2017-55). K.H.W. is supported by the Science and Technology Development Fund of Macau S.A.R. (FDCT; 085/2014/A2). L.E.C. is supported by the Canadian Institutes of Health Research Operating Grants (MOP-86452 and MOP119520), the Natural Sciences and Engineering Council (NSERC) of Canada Discovery Grants (06261 and 462167), and an NSERC E.W.R. Steacie Memorial Fellowship (477598).

\section{Author contributions}

J.L.X., T.K., M.F.M., D.J.K., C.B., B.J.A., A.S., K.H.W., N.R. and L.E.C. designed the experiments. J.L.X., B.T.G., J.D.L.C.D., K.T., M.D.L., T.K., A.S. and N.R. performed the experiments. L.Q., Z.M., K.T. and K.H.W. performed the RNA PolII ChIP experiments and data analysis. J.R.K., J.T. and M.F.M. performed AP and LC-MS analysis. J.L.X., N.R. and L.E.C. wrote the manuscript.

\section{Additional information}

Supplementary Information accompanies this paper at doi:10.1038/s41467-017-00547-y.

Competing interests: The authors declare no competing financial interests.

Reprints and permission information is available online at http://npg.nature.com/ reprintsandpermissions/

Publisher's note: Springer Nature remains neutral with regard to jurisdictional claims in published maps and institutional affiliations.

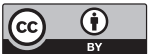

Open Access This article is licensed under a Creative Commons Attribution 4.0 International License, which permits use, sharing, adaptation, distribution and reproduction in any medium or format, as long as you give appropriate credit to the original author(s) and the source, provide a link to the Creative Commons license, and indicate if changes were made. The images or other third party material in this article are included in the article's Creative Commons license, unless indicated otherwise in a credit line to the material. If material is not included in the article's Creative Commons license and your intended use is not permitted by statutory regulation or exceeds the permitted use, you will need to obtain permission directly from the copyright holder. To view a copy of this license, visit http://creativecommons.org/ licenses/by/4.0/.

(C) The Author(s) 2017 